\title{
On the Equivalence of Causal Propagators of the Dirac Equation in Vacuum-Destabilising External Fields
}

\author{
Jonathan J. Beesley ${ }^{1}$ (C)
}

Received: 8 July 2021 / Accepted: 1 January 2022 / Published online: 10 February 2022

(c) The Author(s) 2022

\begin{abstract}
In QED, an external electromagnetic field can be accounted for non-perturbatively by replacing the causal propagators used in Feynman diagram calculations with Green's functions for the Dirac equation under the external field. If the external field destabilises the vacuum, then it is a difficult problem to determine which Green's function is appropriate, and multiple approaches have been developed in the literature whose equivalence, in many cases, is not clear. In this paper, we demonstrate for a broad class of external fields that includes all that act for a finite time, that four Green's functions used in the literature are equivalent: Schwinger's "propertime" propagator; the "causal propagator" used in the "Bogoliubov transformation" method based on the canonical quantization of the field operator; and two defined using analytic continuation from complexified parameters. To do so, we formulate Schwinger's "proper-time quantum mechanics" as a Schrödinger wave mechanics, and use this to re-derive Schwinger's expression for the propagator as a statement relating solutions of the inhomogeneous Dirac equation to those of the inhomogeneous "proper-time Dirac equation". This is done by constructing direct integral spectral decompositions of the Hamiltonians of both equations, and deriving a form for solutions of the inhomogeneous Dirac equation in terms of these decompositions. We then show that all four propagators return solutions of the inhomogeneous Dirac equation that satisfy the same boundary condition, which under a physically reasonable assumption is sufficient to specify the solution uniquely.
\end{abstract}

Keywords Quantum electrodynamics · External field · Unstable vacuum · Propagators $\cdot$ Dirac equation $\cdot$ Proper-time

Jonathan J. Beesley

jjb17@ic.ac.uk

1 Blackett Laboratory, Imperial College, London SW7 2AZ, UK 


\section{Introduction}

The approach of Furry and Feynman [1, 2] to account for an external electromagnetic field in quantum electrodynamics non-perturbatively is to replace causal propagators of the free particle Dirac equation in Feynman diagram calculations with "causal propagators" for the Dirac equation under an external field. This method, though, is only unambiguous if the field is such that, over the course of the scattering event, positive energy states stay as positive energy and negative stay as negative energy. Physically, this is the case if the vacuum is stable under the external field. When we generalise to the case of a vacuum-destabilising external field, such as a constant electric field, two related problems arise: it is not clear what propagator is appropriate, and the interpretation of the "vacuum" becomes problematic. We address the former problem in this paper.

Multiple approaches have been developed to the problem of QED in a vacuumdestabilising external field [3]. We are largely interested in two of the most widely used. First, Schwinger developed a "proper-time" technique for calculating a propagator and the effective action [4]. This was generalised by DeWitt to account for curved spacetimes [5], and by later authors for the general gauge theory [6], as is reviewed [7]. The effective action approach to QED in a vacuum-destabilising field has continued to make heavy use of the proper-time method [8]. Further, the "stringinspired worldline formalism" [9] approach to effective actions has had success in application to nonperturbative particle production in QED [10, 11]. The worldline formalism bears a striking resembles to Schwinger's proper-time method, though is carried out with both Euclidean coordinate-time and Euclidean proper-time.

Second, the "Bogoliubov transform" method based on the canonical quantization of the bispinor field operator [12-15] represents an especially systematic account of QED in a vacuum-destabilising field. It is summarised in [16], and we shall refer to the method from here on as being that of "FGS" after this work's authors. FGS introduce many propagators, associated with electrons and positrons defined with respect to different vacua. It is demonstrated by explicit calculation in Chap. 6 of [16] that their "causal propagator" is equivalent to Schwinger's proper-time representation of the propagator in the case of a constant field superimposed with a plane wave. There is a "strong belief" [17] that the two propagators are equivalent in the general case, but no proof. In this paper we demonstrate equivalence for a broad class of external fields which includes all that act for a finite time.

We do so by considering the Green's function as an operator, $\hat{G}$, on source profiles, $J(x)$, that returns solutions to the inhomogeneous Dirac equation,

$$
\begin{gathered}
\psi(x)=[\hat{G} J](x) \\
\left.\left(i \gamma^{\mu}\left(\partial_{\mu}+i e A_{\mu}\right)\right)-m_{0}\right) \psi(x)=-J(x) .
\end{gathered}
$$

We shall from here on refer to Eq. 2 as the "inhomogeneous spacetime Dirac equation", to distinguish it from the proper-time equation defined in Sect. 2.1. We restrict attention to the case that $J(x)$ is supported only on a finite time, between $t_{\text {in }}<0$ and $t_{\text {out }}>0$. (The assumption that such an operator defines the Green's function well is discussed in Sect. 5.3.1.) We then impose the condition that before the time $t_{\text {in }}<0$ 
and after time $t_{\text {out }}>0$ there is no electric field, and that the gauge field is constant in time. This implies that it can be written with the following restricted dependencies,

$$
A_{\mu}(x)= \begin{cases}\left(A_{0}^{\text {out }}, \mathbf{A}^{\text {out }}(\mathbf{x})\right), & t \geq t_{\text {out }} \\ \left(A_{0}^{\text {in }}, \mathbf{A}^{\text {in }}(\mathbf{x})\right), & t \leq t_{\text {in }} .\end{cases}
$$

For our purposes, Schwinger's principle result is the operator relation

$$
\hat{G}=i \int_{0}^{\infty} d s e^{-\left(i \hat{H}+m_{0}\right) s},
$$

where

$$
\hat{H}:=-i \gamma^{\mu}\left(\partial_{\mu}+i e A_{\mu}\right)
$$

The bulk of this paper, Sect. 2, is devoted to a re-derivation of Eq. 4 as a statement relating solutions of partial differential equations, followed by the result that $\hat{G} J$ necessarily obeys the "Feynman boundary condition", defined in Sect. 2.2.3. Demonstrating equivalence with Schwinger's propagator (4) is then trivial, as shown in Sect. 4.1. In Sect. 3 we demonstrate that solutions derived using FGS's causal propagator also obey the Feynman boundary condition, and in Sect. 4.2 we demonstrate the same for solutions derived using two different varieties of analytic continuation from complexified parameters. Under an additional assumption discussed in Sect. 2.2.3, which we share with FGS, this boundary condition is sufficient to determine the solution of the inhomogeneous equation, and hence the propagator. We therefore demonstrate the equivalence of four different causal propagators, with caveats discussed in Sect. 5.

This acts as an "interpretation" of Schwinger's proper-time formalism, which could be of independent interest to work involved in interpreting the proper-time quantum mechanics as "physically significant", as is reviewed in [18]. In doing this we have also modernised the formalism in which Schwinger's proper-time quantum mechanics is expressed. Schwinger formulated his proper-time quantum mechanics using Dirac's bra-ket notation, which does not account for the difficulties involved with unbounded operators and their continuous eigenbases [19]. FGS similarly circumvent such issues by assuming a discrete eigenbasis for the Dirac Hamiltonian. This work applies formalisms to Schwinger's proper-time quantum mechanics, such as von Neumann's direct integral [20], which have been applied in rigorous formulations of non-relativistic quantum mechanics to account for unbounded operators [21]. Our argument, though, is not a full rigorous proof, as through Sect. 2 we make a number of mathematical assumptions. As discussed in Sect. 5.3, these assumptions are the sort that uses of the bra-ket formalism frequently make implicitly. Using this formalism has the advantage that it makes mathematical assumptions explicit that the more conventional physics formalism leaves implicit. It therefore acts as an initial step towards formulating Schwinger's proper-time quantum mechanics in the rigorous terms that have been developed for regular quantum mechanics since he wrote. 


\section{The Inhomogeneous Proper-Time Dirac Equation}

\subsection{Basic Definitions}

Define $M$ and $S$ as the manifolds formed by attaching a bispinor to every point in spacetime and space, respectively. We are interested in the Hilbert spaces defined by their representation as square-integrable functions on $M$ and $S$,

$$
\mathcal{H}_{\text {P.T. }} \cong L^{2}\left(M, d^{4} x\right), \quad \mathcal{H}_{\text {S.T. }} \cong L^{2}\left(S, d^{3} x\right),
$$

where "P.T." stands for "proper-time" and "S.T." stands for "Spacetime". Denote the inner products on these spaces $(\varphi, \phi)_{\text {P.T. }}$ and $(\varphi, \phi)_{\text {S.T. }}$. There are three basic operators that we consider: $\hat{H}$ on $\mathcal{H}_{\text {P.T. }}(5)$ and two operators on $\mathcal{H}_{\text {S.T. }}$,

$$
\begin{gathered}
\hat{\Pi}^{\mathrm{in} / \text { out }}:=\gamma^{i}\left(\partial_{i}+i e A_{i}^{\mathrm{in} / \text { out }}\right) \\
\hat{\mathscr{H}}_{m}^{\mathrm{in} / \text { out }}:=\gamma^{0}\left(m-i \hat{\Pi}^{\mathrm{in} / \text { out }}\right)+e A_{0}^{\mathrm{in} / \text { out }} .
\end{gathered}
$$

The operator (8) is the "Dirac Hamiltonian" for times $t \leq t_{\text {in }}$ or $t \geq t_{\text {out }}$, since at these times the homogeneous spacetime Dirac equation with mass $m$ can be written on elements of $C^{1}\left(\mathbb{R} ; \mathcal{H}_{\text {S.T. }}\right)$ as

$$
\left(i \partial_{t}-\hat{\mathscr{H}}_{m}^{\mathrm{jn} / \text { out }}\right) \psi(x)=0 .
$$

In Sects. 2.2 and 2.3 we construct representations of, respectively, $\mathcal{H}_{\text {S.T. }}$ and $\mathcal{H}_{\text {P.T. }}$ in which $\hat{\mathscr{H}}_{m}^{\text {in/out }}$ and $\hat{H}$ are represented by multiplication operators. To do this, we use the "direct integral" version of the spectral theorem, as presented in e.g. [21]. If $\hat{A}$ is a self-adjoint operator, then we denote its spectrum by $\sigma(\hat{A})$, its corresponding representation of the Hilbert space by $\mathcal{H}_{\hat{A}}$, and the unitary transformation from the abstract space to $\mathcal{H}_{\hat{A}}$ by $\hat{U}_{\hat{A}}$. The spectral theorem tells us that that there exists a measure, $\mu_{\hat{A}}$, on $\sigma(\hat{A})$, such that

$$
\mathcal{H}_{\hat{A}}:=\int_{\sigma(\hat{A})}^{\oplus} d \mu_{\hat{A}}(\alpha) \mathcal{H}_{\hat{A}}^{\alpha}, \quad[\hat{A} \varphi](\alpha)=\alpha \varphi(\alpha) .
$$

Elements $\varphi \in \mathcal{H}_{\hat{A}}$ are sections on the spectrum with values in the generalised subspaces, $\varphi(\alpha \in \sigma(\hat{A})) \in \mathcal{H}_{\hat{A}}^{\alpha}$. We write the product on these subspaces as $(a, b)_{\alpha}$, and the Hilbert space product is represented by

$$
(\varphi, \phi)=\int_{\sigma(\hat{A})} d \mu_{\hat{A}}(\alpha)(\varphi(\alpha), \phi(\alpha))_{\alpha} .
$$

$\hat{\Pi}^{\text {in/out }}$ and $\hat{H}^{2}$ are symmetric. We assume that they are also self-adjoint, which means we assume that their domain equals the domain of their adjoint. This allows us to use the spectral theorem on them directly, and we then use their spectral representations to construct those of $\hat{\mathscr{H}}_{m}^{\text {jp/out }}$ and $\hat{H}$. The relationship between these 
representations is examined in Sects. 2.4 and 2.5. In Sect. 2.6 these results are used to analyse the relationship between the solutions of the inhomogeneous spacetime Dirac equation (2) and the inhomogeneous proper-time Dirac equation, defined on elements of $C^{1}\left(\mathbb{R} ; \mathcal{H}_{\text {P.T. }}\right)$,

$$
\left(i \partial_{\tau}-\hat{H}\right) \psi(x ; \tau)=-J(x ; \tau) .
$$

We use the convention that the same symbol is used to represent the same vector as it appears in different representations of the same Hilbert space, with the representation being distinguished by the argument of the vector, i.e. if a vector in the abstract space is written as $\varphi \in \mathcal{H}$ then $\left[\hat{U}_{\hat{A}} \varphi\right](\alpha)=\varphi(\alpha)$. We will also write vectors in Hilbert spaces defined as direct sums, $\mathcal{H}_{A} \oplus \mathcal{H}_{B}$, as column vectors. The convention we use is that the top row of the column contains the vector in the space on the left side of the sum, i.e.

$$
\psi=\left[\begin{array}{l}
\phi \\
\varphi
\end{array}\right], \quad \psi \in \mathcal{H}_{A} \oplus \mathcal{H}_{B}, \phi \in \mathcal{H}_{A}, \varphi \in \mathcal{H}_{B}
$$

\subsection{A Representation of $\mathcal{H}_{\text {S.r. }}$ in Which the Dirac Hamiltonian is Diagonal}

\subsubsection{Construction of the Representation}

As discussed in Sect. 2.1, we can define a direct integral representation of $\mathcal{H}_{\text {S.T. }}$ on which $\hat{\Pi}^{\text {in/out }}$ is represented by a multiplication operator,

$$
\mathcal{H}_{\text {in } / \text { out }, \hat{\Pi}}:=\int_{\sigma\left(\hat{\Pi}^{\mathrm{in} / o u t}\right)}^{\oplus} d \mu_{\mathrm{in} / \text { out }}(\Pi) \mathcal{H}_{\mathrm{in} / \text { out }}^{\Pi} .
$$

Because $\gamma^{0}$ is a bounded operator that anticommutes with $\hat{\Pi}^{\text {in/out }}$, it can be represented as a transformation between the generalised subspaces, written in the form $\left(\gamma^{0} \phi\right)(\Pi)=\hat{\gamma}_{\text {in } / \text { out }}^{0}(-\Pi) \phi(-\Pi)$, where $\hat{\gamma}^{0}(\Pi): \mathcal{H}_{\mathrm{in} / \text { out }}^{\prime \prime} \rightarrow \mathcal{H}_{\mathrm{in} / \text { out }}^{-1}$. Since $\left(\gamma^{0}\right)^{2}=1, \hat{\gamma}_{\mathrm{in} / \text { out }}$ ${ }^{0}(\Pi) \hat{\gamma}_{\text {in } / \text { out }}^{0}(-\Pi)=1$. Since this is an invertible unitary transformation, we know from its existence that $\Pi$ is in the point spectrum iff $-\Pi$ is, that $\Pi$ is in the continuous spectrum iff $-\boldsymbol{\Pi}$ is, and that $\operatorname{dim}\left(\mathcal{H}_{\text {in } / \text { out }}^{\Pi}\right)=\operatorname{dim}\left(\mathcal{H}_{\text {in } / \text { out }}^{-\Pi}\right)$. We can therefore choose the measure to be such that we can define a new representation $\mathcal{H}_{\text {in/out },|\hat{\Pi}|}$,

$$
\mathcal{H}_{\text {in } / \text { out },|\hat{\Pi}|}:=\int_{\sigma\left(\hat{\Pi}^{\text {in/out }}\right)>0}^{\oplus} d \mu_{\text {in/out }}(\Pi) \mathcal{H}_{\text {in/out }}^{\Pi} \oplus \mathcal{H}_{\text {in/out }}^{-\Pi}+\mu_{\text {in/out }}(\{0\}) \mathcal{H}_{\text {in/out }}^{0} .
$$

We write $\hat{U}_{|\hat{\Pi}|}^{\text {in/out }}$ for the unitary transformation from $\mathcal{H}_{\text {P.T. }}$ to $\mathcal{H}_{\text {in/out }, \hat{\Pi} \mid \text {, and }}$ $(a, b)_{\Pi \oplus-\Pi}$ for the product on the generalised subspaces. We write sections in this representation as $\phi^{\text {in/out }}(\Pi)$, with the superscript included to distinguish the representation since in this case the usual practice of distinguishing by the argument would be ambiguous.

Now consider the operators on $\mathcal{H}_{\text {in/out }}^{\Pi} \oplus \mathcal{H}_{\text {in/out }}^{-\Pi}$, for $m \neq 0, m \neq \pm i \Pi$, 


$$
\hat{P}_{\text {in/out }, \Pi, m}^{ \pm}: \quad\left[\begin{array}{l}
a \\
b
\end{array}\right] \mapsto \frac{1}{2}\left[\begin{array}{c}
a \pm \frac{\sqrt{m^{2}+\Pi^{2}}}{m-i \Pi} \hat{\gamma}_{\text {in/out }}^{0}(-\Pi) b \\
b \pm \frac{\sqrt{m^{2}+\Pi^{2}}}{m+i \Pi} \hat{\gamma}_{\text {in/out }}^{0}(\Pi) a .
\end{array}\right]
$$

and on $\mathcal{H}_{\text {in/out }}^{0}$,

$$
\hat{P}_{\text {in/out }, 0, m}^{ \pm}: \quad a \mapsto \frac{1}{2}\left(1 \pm \hat{\gamma}^{0}(0)\right) a .
$$

We neglect the $\Pi \neq 0, m= \pm i \Pi$ case, where the operator $\hat{\mathscr{H}}_{m}^{\text {inout }}$ is not diagonalizable. We argue in Sect. 5.3.2 that the assumption that this will not affect our overall argument amounts to a minimal assumption on the spectrum of $\hat{H}$ and $\hat{\Pi}^{\text {in/out }}$. These operators obey $\left(\hat{P}_{\text {in/out }, \Pi, m}^{ \pm}\right)^{2}=\hat{P}_{\text {in } / \text { out }, \Pi, m}^{ \pm}$and $1=\sum_{ \pm} \hat{P}_{\text {in/out }, \Pi, m}^{ \pm}$. They are therefore a complete set of projection operators, and we can define their target spaces as $\mathcal{H}_{\text {in/out }}^{\Pi, m, \pm}$, with $\mathcal{H}_{\text {in/out }}^{\Pi} \oplus \mathcal{H}_{\text {in/out }}^{-\Pi}=\mathcal{H}_{\text {in/out }}^{\Pi, m,+} \oplus \mathcal{H}_{\text {in/out }}^{\Pi, m,-}$. Therefore, for every $m \in \mathbb{C}$, there exists a decomposition of every element $\phi \in \mathcal{H}_{\text {S.T. }}, \phi^{\text {in/out }}(\Pi) \in \mathcal{H}_{\text {in/out }}^{\Pi} \oplus \mathcal{H}_{\text {in/out }}^{-\Pi}$,

$$
\begin{gathered}
\phi(\Pi)=\sum_{ \pm} \phi(\Pi)_{m}, \quad\left[\hat{\mathscr{H}}_{m}^{\text {in }} \phi\right](\Pi)=\sum_{ \pm} E_{ \pm}^{\text {in }}(m, \Pi)_{ \pm} \phi_{m}(\Pi) \\
\phi(\Pi)=\sum_{ \pm}{ }^{ \pm} \phi_{m}(\Pi), \quad\left[\hat{\mathscr{H}}_{m}^{\mathrm{ut}} \phi_{m}\right](\Pi)=\sum_{ \pm} E_{ \pm}^{\mathrm{out}}(m, \Pi)^{ \pm} \phi_{m}(\Pi),
\end{gathered}
$$

where

$$
\begin{gathered}
{ }_{ \pm} a_{m}:=\hat{P}_{\mathrm{in}, \Pi, m}^{ \pm} a, \quad{ }^{ \pm} a_{m}:=\hat{P}_{\mathrm{out}, \Pi, m}^{ \pm} a \\
E_{ \pm}^{\mathrm{in} / \text { out }}(m, \Pi):= \pm \sqrt{m^{2}+\Pi^{2}}+e A_{0}^{\mathrm{in} / \text { out }} .
\end{gathered}
$$

\subsubsection{The Time-Translation Operator}

We can define a time-translation operator for the homogeneous spacetime Dirac equation with mass $m$ on $\mathcal{H}_{\text {in/out, }|\hat{\Pi}|}$,

$$
\begin{gathered}
{\left[\hat{T}_{m}\left(t, t^{\prime}\right) \phi\right]^{\text {in }}(\Pi)=\sum_{ \pm}{ }_{ \pm} \phi_{m}(\Pi) \exp \left(-i E_{ \pm}^{\text {in }}(m, \Pi)\left(t-t^{\prime}\right)\right), \quad t, t^{\prime} \leq t_{\text {in }}} \\
{\left[\hat{T}_{m}\left(t, t^{\prime}\right) \phi\right]^{\text {out }}(\Pi)=\sum_{ \pm}{ }^{ \pm} \phi_{m}(\Pi) \exp \left(-i E_{ \pm}^{\text {out }}(m, \Pi)\left(t-t^{\prime}\right)\right), t, t^{\prime} \geq t_{\text {out }} .}
\end{gathered}
$$

When acting on the domain of $\hat{\mathscr{H}}_{m}^{\text {in }}$ in $\mathcal{H}_{\text {S.T. }}$ it returns the unique solution for the spacetime Dirac equation with mass $m$ in $C^{1}\left(\left(-\infty, t_{\text {in }}\right] ; \mathcal{H}_{\text {S.T. }}\right)$ or $C^{1}\left(\left[t_{\text {out }}, \infty\right) ; \mathcal{H}_{\text {S.T. }}\right)$ that equals the input state at $t^{\prime}$. The fact that it returns a solution of the Dirac equation with mass $m$ and leaves it invariant if $t=t^{\prime}$ is clear from inspection. That the solution it gives is unique and that it is necessarily in the Hilbert space is shown in Appendix 2. If $m$ is real then $\hat{T}_{m}\left(t, t^{\prime}\right)$ is unitary. 


\subsubsection{The Bipartition of $\mathcal{H}_{\text {S.T. }}$ and Feynman Boundary Conditions}

Define $\hat{P}_{\text {in/out }, m}^{ \pm}$as the operator on $\mathcal{H}_{\text {S.T. }}$ induced by the $\hat{P}_{\text {in/out }, \Pi, m}^{ \pm}$operators on the generalised subspaces, $\hat{P}_{\text {in/out }, m}^{ \pm}: \phi(\Pi) \mapsto \hat{P}_{\text {in/out, } \Pi, m}^{ \pm} \phi(\Pi)$. We can then define the target spaces of $\hat{P}_{\text {in }, m}^{ \pm}$as ${ }_{ \pm} \mathcal{H}_{\mathrm{S} \text {.T. }}^{m}$, and the target spaces of $P_{\text {out }, m}^{ \pm}$as ${ }^{ \pm} \mathcal{H}_{\mathrm{S} \text {.T. }}^{m}$, such that

$$
\mathcal{H}_{\text {S.T. }}={ }_{+} \mathcal{H}_{\text {S.T. }}^{m} \oplus{ }_{-} \mathcal{H}_{\text {S.T. }}^{m}={ }^{+} \mathcal{H}_{\text {S.T. }}^{m} \oplus{ }^{-} \mathcal{H}_{\text {S.T. }}^{m}
$$

Note that this bipartition is orthogonal if $m$ is real, and

$$
{ }_{ \pm} \phi_{m} \in{ }_{ \pm} \mathcal{H}_{\mathrm{S} . \mathrm{T} .}^{m}, \quad{ }^{ \pm} \phi_{m} \in{ }^{ \pm} \mathcal{H}_{\mathrm{S} \text { S.T. }}^{m} .
$$

Definition (Feynman boundary condition) An element of $C\left(L ; \mathcal{H}_{\text {S.T. }}\right)$, where $t_{\text {in }}, t_{\text {out }} \in L \subseteq \mathbb{R}$, is said to obey the "Feynman boundary condition (with mass $m$ )" if and only if it has 0 component in ${ }_{+} \mathcal{H}_{\text {S.T. }}^{m}$ at $t_{\text {in }}$ and 0 component in ${ }^{-} \mathcal{H}_{\text {S.T. }}^{m}$ at $t_{\text {out }}$.

The name is chosen as the boundary condition is a natural generalisation of that discussed by Feynman for the free particle causal propagator [2]. It is a straightforward consequence of the linearity of the boundary condition that it uniquely determines a solution of the inhomogeneous spacetime Dirac equation if and only if there exists no nontrivial solution of the homogeneous spacetime Dirac equation that satisfies the boundary condition. A closely related condition is discussed by FGS [16, Chap. 2.4]. They demand that the determinant of the transformation matrix between the basis of negative energy in-states and negative energy out-states is non-zero. This would be the case if and only if there are no vectors in the space of negative energy in-states which transform to vectors with no components in the negative energy out-space - i.e. there are no nontrivial solutions to the homogeneous spacetime Dirac equation which satisfy the Feynman boundary condition. FGS introduce this as a condition for the Fock spaces formed by the action of the creation operators on the in and out vacua to coincide. The physical implications of this condition being violated are unclear, but probably significant: it seems to correspond to a situation where the vacuum states at the in and out times are too radically different to be described in terms of particle perturbations of each other, and hence the whole approach to externalfield QED that thinks in terms of perturbative particle processes expanded about the transition from vacuum to vacuum breaks down. Therefore, in physical situations where any of the approaches to external-field QED being considered in this paper are appropriate, this condition ought to be satisfied.

\subsection{A Representation of $\mathcal{H}_{\text {P.T. }}$ in Which $\hat{\boldsymbol{H}}$ is Diagonal}

As discussed in Sect. 2.1, we can define a direct integral representation of $\mathcal{H}_{\text {P.T. }}$ on which $\hat{H}^{2}$ is represented by a multiplication operator,

$$
\mathcal{H}_{\hat{H}^{2}}:=\int_{\sigma\left(\hat{H}^{2}\right)}^{\oplus} d \mu_{\hat{H}^{2}}\left(\lambda^{2}\right) \mathcal{H}_{\hat{H}^{2}}^{\lambda^{2}} .
$$


Consider the representation of $\hat{H}$ on this space. Since it commutes with $\hat{H}^{2}$, it must be able to be represented by an operator $\hat{H}\left(\lambda^{2}\right)$ on every $\mathcal{H}_{\hat{H}^{2}}^{\lambda^{2}}$ space, and since $\left(\hat{H}\left(\lambda^{2}\right)\right)^{2}=\lambda^{2}$, we know that if $\lambda \neq 0$ then $\hat{H}\left(\lambda^{2}\right) / \lambda$ is defined everywhere on this space [22]. We can therefore define the operators, for $\lambda^{2} \neq 0$,

$$
\hat{P}_{\lambda} a:=\frac{1}{2}\left(1+\frac{\hat{H}}{\lambda}\right) a .
$$

These obey $\left(\hat{P}_{\lambda}\right)^{2}=\hat{P}_{\lambda}$ and $1=\hat{P}_{\lambda}+\hat{P}_{-\lambda}$. They are therefore a complete set of projection operators, and we can define their target spaces as $\mathcal{H}_{\hat{H}}^{\lambda}$ such that

$$
\mathcal{H}_{\hat{H}^{2}}^{\lambda^{2}}=\mathcal{H}_{\hat{H}}^{\lambda} \oplus \mathcal{H}_{\hat{H}}^{-\lambda}
$$

This bipartition is orthogonal if $\lambda$ is real. Define also $\hat{P}_{0}:=1$. We can then define $\mathcal{H}_{\hat{H}}$ and $\hat{U}_{\hat{H}}: \mathcal{H}_{\text {P.T. }} \rightarrow \mathcal{H}_{\hat{H}}$ by

$$
\mathcal{H}_{\hat{H}}:=\int_{\sigma(\hat{H})}^{\oplus} d \mu_{\hat{H}}(\lambda) \mathcal{H}_{\hat{H}}^{\lambda}, \quad\left[\hat{U}_{\hat{H}} \phi\right](\lambda)=\hat{P}_{\lambda}\left[\hat{U}_{\hat{H}^{2}} \phi\right]\left(\lambda^{2}\right)
$$

where $\mu_{\hat{H}}$ is the push-forward of $\mu_{\hat{H}^{2}}$ by $\lambda=\operatorname{sign}(\lambda) \sqrt{\lambda^{2}}$. (Considering $\lambda^{2}$ as an independent variable.) Note that since $(\sigma(\hat{H}))^{2}=\sigma\left(\hat{H}^{2}\right), \sigma(\hat{H})$ is confined to the real and imaginary axes of the complex plane. It directly follows from Eqs. 24 and 26 that $\hat{H}$ is represented in $\mathcal{H}_{\hat{H}}$ as a multiplication operator, $\hat{H}: \phi(\lambda) \mapsto \lambda \phi(\lambda)$. To derive the representation of the Hilbert-space product in $\mathcal{H}_{\hat{H}}$, first define

$$
\bar{\varphi}(\lambda):=\frac{1}{2}\left(1+\frac{\hat{H}^{\dagger}}{\lambda^{*}}\right) \varphi(\lambda) .
$$

This gives

$$
\begin{aligned}
(\varphi, \phi)_{\mathrm{P} . \mathrm{T} .} & =\int_{\sigma\left(\hat{H}^{2}\right)} d \mu_{\hat{H}^{2}}\left(\lambda^{2}\right) \sum_{ \pm}\left(\bar{\varphi}\left( \pm \sqrt{\lambda^{2}}\right), \phi\left( \pm \sqrt{\lambda^{2}}\right)\right)_{ \pm \sqrt{\lambda^{2}}} \\
& =\int_{\sigma(\hat{H})} d \mu_{\hat{H}}(\lambda)(\bar{\varphi}(\lambda), \phi(\lambda))_{\lambda}
\end{aligned}
$$

where $(a, b)_{\lambda}$ is the inner product of $\mathcal{H}_{\hat{H}}^{\lambda}$. In the last equality we have moved the sum over \pm outside the integral, which we must assume to be possible.

\subsection{Generalised Eigenvectors of $\hat{H}$}

Consider a vector in one of the generalised subspaces that makes up the direct integral $\mathcal{H}_{\hat{H}}, a \in \mathcal{H}_{\hat{H}}^{\lambda_{0}}$. If $\lambda_{0}$ is in the point spectrum, then $a$ is a true eigenvector of $\hat{H}$. If $\lambda_{0}$ is in the continuous spectrum, though, then this "generalised eigenvector" is not a vector in the Hilbert space proper. Whether $\lambda_{0}$ is in the continuous or point spectrum, it can be seen as a representation-independent object by its action as a linear functional, 


$$
\langle\varphi, a\rangle:=\left(\bar{\varphi}\left(\lambda_{0}\right), a\right)_{\lambda_{0}} .
$$

This functional action is well-defined on any $\varphi \in \mathcal{H}_{\hat{H}}$ for which $\bar{\varphi}$ is of finite magnitude at $\lambda=\lambda_{0}$. This includes the whole Hilbert space iff $\lambda_{0}$ is in the point spectrum.

These generalised eigenvectors are functional solutions of the homogeneous spacetime Dirac equation with mass $m=-\lambda_{0}$. We assume that they are able to be chosen to have some desirable properties. First, we assume that they are classical solutions at $t \leq t_{\text {in }}$ and $t \geq t_{\text {out }}$. Specifically, for every $a \in \mathcal{H}_{\hat{H}}^{\lambda}$, there exists $a\left(t \leq t_{\text {in }}\right) \in C^{1}\left(\left(-\infty, t_{\text {in }}\right] ; \mathcal{H}_{\text {S.T. }}\right)$ and $a\left(t \geq t_{\text {out }}\right) \in C^{1}\left(\left[t_{\text {out }}, \infty\right) ; \mathcal{H}_{\text {S.T. }}\right)$, such that if $\langle\varphi, a\rangle$ exists then,

$$
\lim _{n \rightarrow \infty}\left\langle\delta_{t}^{(n)} \varphi, a\right\rangle=(\varphi(t), a(t))_{\mathrm{S} . \mathrm{T}}, \quad t \leq t_{\text {in }} \quad \text { or } \quad t \geq t_{\text {out }},
$$

where $\delta_{t}^{(n)}$ is a sequence of $\mathbb{C}$-valued functions that act as a nascent delta function centred on $t$ as $n \rightarrow \infty$. This means that we can define a mapping from every $\mathcal{H}_{\hat{H}}^{\lambda}$ to $\mathcal{H}_{\text {S.T. }}$ for every time $t \leq t_{\text {in }}$ or $t \geq t_{\text {out }}, a \mapsto a(t)$, and that for times $t, t^{\prime} \leq t_{\text {in }}$ or $t, t^{\prime} \geq t_{\text {out }}$ these are related by $a(t)=\hat{T}_{-\lambda}\left(t, t^{\prime}\right) a\left(t^{\prime}\right)$ as defined in Eqs. 20a and 20b.

We need two other results. First, it follows straightforwardly from Eqs. 20a and $20 \mathrm{~b}$ and $(a, a)_{\text {P.T. }}=\int_{-\infty}^{\infty} d t(a(t), a(t))_{\text {S.T. }}$ that for $a \in \mathcal{H}_{\hat{H}}^{\lambda}$ to have finite $\mathcal{H}_{\text {P.T. }}$-norm, $E_{ \pm}^{\text {in }}(\lambda, \Pi)$ must have positive imaginary component for every non-zero ${ }_{ \pm} a_{-\lambda}\left(\Pi ; t_{\text {in }}\right)$, and $E_{ \pm}^{\text {out }}(\lambda, \Pi)$ must have negative imaginary component for every non-zero ${ }^{ \pm} a_{-\lambda}\left(I \bar{\Pi} ; t_{\text {out }}\right)$. A true eigenstate of $\hat{H}$ cannot have a component of real energy. We want a similar though less strict restriction on the generalised eigenstates, which we assume rather than demonstrate. We assume that the generalised eigenvectors $a \in \mathcal{H}_{\hat{H}}^{\lambda}$ do not grow exponentially. This would necessarily be true if the external field is smooth with all derivatives polynomially bounded, as then $\hat{H}$ leaves the Schwartz class invariant, and hence we could use the nuclear spectral theorem to determine that our eigenstates are tempered distributions [23].

\subsection{Projecting a Vector in $\mathcal{H}_{\text {P.т. }}$ onto a Vector in $\mathcal{H}_{\text {S.T. }}$}

Since $\mathcal{H}_{\text {P.T. }} \cong L^{2}\left(\mathbb{R} ; \mathcal{H}_{\text {S.T. }}\right)$, for any $\phi \in \mathcal{H}_{\text {P.T. }}$ we can assign, for every $t \in \mathbb{R}$, $\phi(t) \in \mathcal{H}_{\text {S.T. }}$. This means of projecting a Hilbert-space state onto a time must be consistent with the projection of the generalised eigenvectors just discussed. Therefore,

$$
\begin{aligned}
\lim _{n \rightarrow \infty}\left(\varphi \delta_{t}^{(n)}, \phi\right)_{\text {P.T. }} & =(\varphi(t), \phi(t))_{\text {S.T. }}=\int_{\sigma(\hat{H})} d \mu_{\hat{H}}(\lambda) \lim _{n \rightarrow \infty}\left\langle\varphi(\lambda) \delta_{t}^{(n)}, \phi(\lambda)\right\rangle \\
& =\int_{\sigma(\hat{H})} d \mu_{\hat{H}}(\lambda)(\bar{\varphi}(t), \phi(\lambda ; t))_{\text {S.T. }} .
\end{aligned}
$$

Working in the $\mathcal{H}_{\text {in/out, }|\hat{\Pi}|}$ this becomes 


$$
\begin{aligned}
& \int_{\sigma(\hat{\Pi} \text { in/out }) \geq 0} d \mu_{\text {in } / \text { out }}(\Pi)\left(\varphi^{\text {in } / \text { out }}(\Pi), \phi^{\text {in } / \text { out }}(\Pi ; t)\right)_{\Pi \oplus-\Pi} \\
& =\int_{\sigma(\hat{H})} d \mu_{\hat{H}}(\lambda) \int_{\sigma(\hat{\Pi} \text { in/out }) \geq 0} d \mu_{\text {in } / \text { out }}(\Pi)\left(\varphi^{\text {in } / \text { out }}(\Pi),\right. \\
& \left.\phi^{\text {in } / \text { out }}(\Pi ; \lambda ; t)\right)_{\Pi \oplus-\Pi} .
\end{aligned}
$$

Assuming that we can change the order the integrals and the implicit sum over the basis sections of $\mathcal{H}_{\text {in/out },|\hat{I}|}$, this gives

$$
\phi^{\text {in } / o u t}(\Pi ; t)=\int_{\sigma(\hat{H})} d \mu_{\hat{H}}(\lambda) \phi^{\text {in } / o u t}(\Pi ; \lambda ; t) .
$$

The primary aim of this subsection is to convert the RHS of this equation into an inverse Fourier transform for times $t \leq t_{\text {in }}$ and $t \geq t_{\text {out }}$. Call $I_{\text {in/out }}^{ \pm}$the open vertical half-line in the upper (+ case) or lower ( - case) complex half-plane that has $e A_{0}^{\text {in/out }}$ as its finite limit point. Call $I_{\text {in/out }}$ the verticle line in the complex plane that intersects the real axis at $e A_{0}^{\text {in/out }}$. Call $i \mathbb{R}$ the imaginary axis, and $i \mathbb{R}^{ \pm}$the open positive/ negative imaginary half-axes. Extend the measure $\mu_{\hat{H}}$ to the whole imaginary and real axes by stipulating $\mu_{\hat{H}}(\rho)=0$ if $\rho \cap \sigma(\hat{H})$ is empty. Then we can use Eqs. 20a and $20 \mathrm{~b}$ to write Eq. 33 as

$$
\begin{aligned}
\phi^{\text {in }}(\Pi ; t) & =\sum_{\xi= \pm} \int_{\mathbb{R} \cup i \mathbb{R}} d \mu_{\hat{H}}(\lambda)_{\xi} \phi_{-\lambda}\left(\Pi ; \lambda ; t^{\prime}\right) \exp \left(-i E_{\xi}^{\text {in }}(\lambda, \Pi)\left(t-t^{\prime}\right)\right) \\
\phi^{\text {out }}(\Pi ; t) & =\sum_{\xi= \pm} \int_{\mathbb{R} \cup i \mathbb{R}} d \mu_{\hat{H}}(\lambda)^{\xi} \phi_{-\lambda}\left(\Pi ; \lambda ; t^{\prime}\right) \exp \left(-i E_{\xi}^{\text {out }}(\lambda, \Pi)\left(t-t^{\prime}\right)\right),
\end{aligned}
$$

where we assume it is possible to bring the sum over \pm outside of the integral. Define

$$
\lambda^{\text {in } / \text { out }}(E, \Pi):=\sqrt{\left(E-e A_{0}^{\text {in } / \text { out }}\right)^{2}-\Pi^{2}},
$$

and

$$
\begin{gathered}
\phi_{\xi}^{\mathrm{in}, \pm}(\Pi ; E ; t):={ }_{\xi} \phi_{\mp \lambda^{\text {in }}(E, \Pi)}\left(\Pi ; \pm \lambda^{\text {in }}(E, \Pi) ; t\right) \\
\phi_{\xi}^{\text {out }, \pm}(\Pi ; E ; t):={ }^{\xi} \phi_{\mp \lambda^{\text {out }}(E, \Pi)}\left(\Pi ; \pm \lambda^{\text {out }}(E, \Pi) ; t\right)
\end{gathered}
$$

such that

$$
\begin{array}{cc}
\phi_{\xi}^{\mathrm{in}, \pm}\left(\Pi ; E_{\xi^{\prime}}^{\mathrm{in}}(\Pi, \lambda) ; t\right)={ }_{\xi} \phi_{-\lambda}(\Pi ; \lambda ; t), \quad \lambda \in \mathbb{R}^{ \pm} \cup i \mathbb{R}^{ \pm} \\
\phi_{\xi}^{\mathrm{out}, \pm}\left(\Pi ; E_{\xi^{\prime}}^{\mathrm{out}}(\Pi, \lambda) ; t\right)={ }^{\xi} \phi_{-\lambda}(\Pi ; \lambda ; t), \quad \lambda \in \mathbb{R}^{ \pm} \cup i \mathbb{R}^{ \pm}
\end{array}
$$


Then, splitting the integrals in Eq. 34 in half, we use Eq. 37 to write them in a form amenable to a change of variables,

$$
\begin{gathered}
\int_{\mathbb{R}^{ \pm} \cup i \mathbb{R}^{ \pm}} d \mu_{\hat{H}}(\lambda) \phi_{\xi}^{\text {in/out }, \pm}\left(\Pi ; E_{\xi}^{\text {in } / \text { out }}(\Pi, \lambda) ; t^{\prime}\right) \exp \left(-i E_{\xi}^{\text {in } / \text { out }}(\Pi, \lambda)\left(t-t^{\prime}\right)\right) \\
=\int_{\left[\mathbb{R}^{\xi}+\left\{e A_{0}^{\text {in } / \text { out }}\right\}\right] \cup I_{\text {in } / \text { out }}^{\xi}} d \mu_{\hat{E}, \Pi}^{\text {in } / \text { out }}(E) \phi_{\xi}^{\text {in } / \text { out }, \pm}\left(\Pi ; E ; t^{\prime}\right) \exp \left(-i E\left(t-t^{\prime}\right)\right),
\end{gathered}
$$

where $\mu_{\hat{E}, \Pi}^{\text {in } / \text { out }}$ is the push-forward of the measure $\mu_{\hat{H}}$ using the mapping $E_{\operatorname{sign}\left(E-e A_{0}^{\text {in } / \text { out }}\right)}^{\text {in } \text { out }}(\Pi, \lambda)$, where $\operatorname{sign}(x)$ returns \pm if $x \in \mathbb{R}^{ \pm} \cup i \mathbb{R}^{ \pm}$. Therefore

$$
\begin{aligned}
& \phi^{\text {in }}(\Pi, t)=\int_{\mathbb{R} \cup I_{\text {in }}^{+}} d \mu_{\hat{E}, \Pi}^{\text {in }}(E)\left(\left[\operatorname { s i g n } \left(E-e A_{0}^{\text {in })]} \phi_{-\lambda^{\text {in }}(E, \Pi)}\left(\Pi ; \lambda^{\text {in }}(E, \Pi) ; t^{\prime}\right)\right.\right.\right. \\
& \left.+_{\left[\operatorname{sign}\left(E-e A_{0}^{\mathrm{in}}\right)\right]} \phi_{\lambda^{\mathrm{in}(E, \Pi)}}\left(\Pi ;-\lambda^{\mathrm{in}}(E, \Pi) ; t^{\prime}\right)\right) \exp \left(-i E\left(t-t^{\prime}\right)\right) \\
& \phi^{\text {out }}(\Pi, t)=\int_{\mathbb{R} \cup I_{\text {out }}^{-}} d \mu_{\hat{E}, \Pi}^{\text {out }}(E)\left(\left[\operatorname{sign}\left(E-e A_{0}^{\text {out }}\right)\right] \phi_{-\lambda^{\text {out }}(E, \Pi)}\left(\Pi ; \lambda^{\text {in }}(E, \Pi) ; t^{\prime}\right)\right. \\
& \left.+{ }^{\left[\operatorname{sign}\left(E-e A_{0}^{\text {out }}\right)\right]} \phi_{\lambda_{\text {out }}(E, \Pi)}\left(\Pi ;-\lambda^{\text {out }}(E, \Pi) ; t^{\prime}\right)\right) \exp \left(-i E\left(t-t^{\prime}\right)\right),
\end{aligned}
$$

where we have used our assumption, discussed at the end of Sect. 2.4, that generalised eigenvectors cannot exponentially grow to eliminate half the integral over the vertical line. Define

$$
\begin{gathered}
\lambda^{\mathrm{u}}(E, \Pi):= \begin{cases}\operatorname{sign}\left(E-e A_{0}^{\text {in }}\right) \sqrt{\left(E-e A_{0}^{\text {in }}\right)^{2}-\Pi^{2}}, & \left(E-e A_{0}^{\text {in }}\right)^{2} \geq \Pi^{2} \\
\sqrt{\left(E-e A_{0}^{\text {in }}\right)^{2}-\Pi^{2}}, & \left(E-e A_{0}^{\text {in }}\right)^{2} \leq \Pi^{2}\end{cases} \\
\lambda^{\mathrm{l}}(E, \Pi):= \begin{cases}\operatorname{sign}\left(E-e A_{0}^{\text {out }}\right) \sqrt{\left(E-e A_{0}^{\text {out }}\right)^{2}-\Pi^{2},} & \left(E-e A_{0}^{\text {out }}\right)^{2} \geq \Pi^{2} \\
-\sqrt{\left(\left(E-e A_{0}^{\text {out }}\right)^{2}-\Pi^{2}\right.}, & \left(E-e A_{0}^{\text {out }}\right)^{2} \leq \Pi^{2},\end{cases}
\end{gathered}
$$

where the " $u$ " and "l" superscript refer to the fact that $\lambda^{\mathrm{u} / 1}$ can be taken as an anlytic function of $E$ on the whole upper/lower half complex plane. Define also

$$
\begin{aligned}
& { }_{ \pm} \phi(\Pi ; E):={ }_{\left[\operatorname{sign}\left(E-e A_{0}^{\mathrm{in}}\right)\right]} \phi_{\mp \lambda^{\mathrm{u}}(E, \Pi)}\left(\Pi ; \pm \lambda^{\mathrm{u}}(E, \Pi) ; t_{\mathrm{in}}\right) \\
& { }^{ \pm} \phi(\Pi ; E):={ }^{\left[\operatorname{sign}\left(E-e A_{0}^{\mathrm{out}}\right)\right]} \phi_{\mp \lambda^{\mathrm{l}}(E, \Pi)}\left(\Pi ; \pm \lambda^{\mathrm{l}}(E, \Pi) ; t_{\mathrm{out}}\right),
\end{aligned}
$$

such that 


$$
\begin{aligned}
\phi^{\text {in }}(\Pi, t) & =\sum_{ \pm} \int_{\mathbb{R} \cup I_{\text {in }}^{+}} d \mu_{\hat{E}, \Pi}^{\text {in }}(E)_{ \pm} \phi(\Pi ; E) \exp \left(-i E\left(t-t_{\text {in }}\right)\right) \\
\phi^{\text {out }}(\Pi, t) & =\sum_{ \pm} \int_{\mathbb{R} \cup I_{\text {out }}^{-}} d \mu_{\hat{E}, \Pi}^{\text {out }}(E)^{ \pm} \phi(\Pi ; E) \exp \left(-i E\left(t-t_{\text {out }}\right)\right) .
\end{aligned}
$$

We proceed, in the rest of this subsection, to convert the integral over imaginary energies to one over real energies, possible by virtue of the assumption that exponential increase is forbidden. This completes the conversion of the RHS of Eq. 33 into an inverse Fourier transform. We need quite a general result, for which we assume that we can express ${ }_{ \pm} \phi(\Pi ; E)$ and ${ }^{ \pm} \phi(\Pi ; E)$ in the form

$$
{ }_{ \pm} \phi(\Pi ; E)=g^{\mathrm{u}}(E){ }_{ \pm} \theta(\Pi ; E), \quad{ }^{ \pm} \phi(\Pi ; E)=g^{1}(E){ }^{ \pm} \theta(\Pi ; E),
$$

where $g^{\mathrm{u} / 1}(E)$ is a complex-valued meromorphic function on the upper/lower halfplanes which is bounded on $I_{\text {in/out }} \cup \mathbb{R}$ and as $|E| \rightarrow \infty$. Note that $g^{\mathrm{u} / \mathrm{l}}(E)$ could depend on \pm and $\Pi$, but since this possible dependence does not affect the following manipulations, we neglect it here. Define

$$
R^{\mathrm{u} / 1}(p, t)[g]:=\sum_{E_{0} \in \text { poles }} \frac{\operatorname{Res}\left(g\left(E_{0}\right)\right)}{E_{0}-p} \exp \left(-i E_{0} t\right),
$$

where the sum runs over poles of $g(E)$ in the upper/lower half plane. Then, by the residue theorem and Jordan's lemma,

$$
\begin{aligned}
& g^{\mathrm{u}}(p) e^{-i p\left(t-t_{\mathrm{in}}\right)}=\int_{\mathbb{R}} d E \frac{g^{\mathrm{u}}(E)}{2 \pi i(E-p)} e^{-i E\left(t-t_{\mathrm{in}}\right)} \\
& -R^{\mathrm{u}}\left(p, t-t_{\text {in }}\right)\left[g^{\mathrm{u}}\right], \quad \operatorname{Im}\{p\}>0 \\
& g^{1}(p) e^{-i p\left(t-t_{\text {out }}\right)}=-\int_{\mathbb{R}} d E \frac{g^{1}(E)}{2 \pi i(E-p)} e^{-i E\left(t-t_{\text {out }}\right)} \\
& -R^{1}\left(p, t-t_{\text {out }}\right)\left[g^{1}\right], \quad \operatorname{Im}\{p\}<0,
\end{aligned}
$$

by closing the $E$ integral in the upper plane for the "in" case and the lower plane for the "out" case. Assuming that we can exchange the order of the integrals along the vertical lines, i.e.

$$
\begin{aligned}
& \int_{I_{\mathrm{in}}^{+}} d \mu_{\hat{E}, \Pi}^{\mathrm{in}}(p) \int_{\mathbb{R}} d E \frac{g^{\mathrm{u}}(E)_{ \pm} \theta(\Pi ; p)}{2 \pi i(E-p)} e^{-i E\left(t-t_{\mathrm{in}}\right)} \\
& =\int_{\mathbb{R}} d E \int_{I_{\mathrm{in}}^{+}} d \mu_{\hat{E}, \Pi}^{\mathrm{in}}(p) \frac{g^{\mathrm{u}}(E)_{ \pm} \theta(\Pi ; p)}{2 \pi i(E-p)} e^{-i E\left(t-t_{\mathrm{in}}\right)}
\end{aligned}
$$


and

$$
\begin{aligned}
& \int_{I_{\text {out }}^{-}} d \mu_{\hat{E}, \Pi}^{\text {out }}(p) \int_{\mathbb{R}} d E \frac{g^{1}(E)^{ \pm} \theta(\Pi ; p)}{2 \pi i(E-p)} e^{-i E\left(t-t_{\text {out }}\right)} \\
& =\int_{\mathbb{R}} d E \int_{I_{\text {out }}^{-}} d \mu_{\hat{E}, \Pi}^{\text {out }}(p) \frac{g^{1}(E)^{ \pm} \theta(\Pi ; p)}{2 \pi i(E-p)} e^{-i E\left(t-t_{\text {out }}\right)} .
\end{aligned}
$$

This allows us to write

$$
\begin{aligned}
\phi^{\text {in }}(\Pi, t)= & \sum_{ \pm} \int_{\mathbb{R}} d E g^{\mathrm{u}}(E)_{ \pm} \check{\theta}(\Pi ; E) \exp \left(-i E\left(t-t_{\text {in }}\right)\right) \\
& -\int_{I_{\text {in }}^{+}} d \mu_{\hat{E}, \Pi}^{\text {in }}(p) R^{\mathrm{u}}\left(p, t-t_{\text {in }}\right)\left[g^{\mathrm{u}}\right]_{ \pm} \theta(\Pi ; p), \quad t \leq t_{\text {in }} \\
\phi^{\text {out }}(\Pi, t)= & \sum_{ \pm} \int_{\mathbb{R}} d E g^{\mathrm{l}}(E)^{ \pm} \check{\theta}(\Pi ; E) \exp \left(-i E\left(t-t_{\text {out }}\right)\right) \\
& -\int_{I_{\text {out }}^{-}} d \mu_{\hat{E}, \Pi}^{\text {out }}(p) R^{\mathrm{l}}\left(p, t-t_{\text {out }}\right)\left[g^{1}\right]^{ \pm} \theta(\Pi ; p), \quad t \geq t_{\text {out }},
\end{aligned}
$$

where,

$$
\begin{gathered}
{ }_{ \pm} \check{\theta}(\Pi ; E):=\rho^{\text {in }}(\Pi ; E){ }_{ \pm} \theta(\Pi ; E)+\int_{I_{\text {in }}^{+}} d \mu_{\hat{E}, \Pi}^{\text {in }}(p) \frac{{ }_{ \pm} \theta(\Pi ; p)}{2 \pi i(E-p)} \\
{ }_{ \pm} \check{\theta}(\Pi ; E):=\rho^{\text {out }}(\Pi ; E)_{ \pm} \theta(\Pi ; E)-\int_{I_{\text {out }}^{-}} d \mu_{\hat{E}, \Pi}^{\text {out }}(p) \frac{{ }_{ \pm} \theta(\Pi ; p)}{2 \pi i(E-p)}
\end{gathered}
$$

and

$$
d \mu_{\hat{E}, \Pi}^{\text {in } / \text { out }}(E)=d E \rho^{\text {in } / \text { out }}(\Pi ; E)
$$

for real $E$, where $\rho^{\text {in/out }}(\Pi ; E) \in[0, \infty)$. This expression for the measure is possible since we know that true eigenvectors of $\hat{H}$ cannot have components with real energy, hence $\mu_{\hat{E}, \Pi}^{\text {in } / \text { out }}$ for real $E$ must be absolutely continuous with respect to the Lebesgue measure.

\subsection{The Differential Equations}

\subsubsection{The Relationship Between Solutions of the Inhomogeneous Spacetime and Proper-Time Dirac Equations}

The inhomogeneous proper-time Dirac Eq. (11) can be written in $\mathcal{H}_{\hat{H}}$ as 


$$
\left(i \partial_{\tau}-\lambda\right) \psi(\lambda ; \tau)=-J(\lambda ; \tau)
$$

Suppose that the complex Fourier transform of $J(\tau)$, with respect to proper-time is well-defined and finite on the positive imaginary axis, and denote it

$$
\tilde{J}(m):=\frac{1}{2 \pi} \int_{\mathbb{R}} d \tau J(\tau) e^{i m \tau}, \quad m \in \mathbb{C} .
$$

Consider

$$
\psi(\lambda ; \tau)= \begin{cases}\int_{-\infty}^{\infty} d m \frac{1}{\lambda-m} \tilde{J}(\lambda ; m) e^{-i m \tau}+2 \pi i \tilde{J}(\lambda ; \lambda) e^{-i \lambda \tau}, & \operatorname{Im}\{\lambda\}>0 \\ P \int_{-\infty}^{\infty} d m \frac{1}{\lambda-m} \tilde{J}(\lambda ; m) e^{-i m \tau}+\pi i \tilde{J}(\lambda ; \lambda) e^{-i \lambda \tau}, & \operatorname{Im}\{\lambda\}=0 \\ \int_{-\infty}^{\infty} d m \frac{1}{\lambda-m} \tilde{J}(\lambda ; m) e^{-i m \tau}, & \operatorname{Im}\{\lambda\}<0 .\end{cases}
$$

Using the Sokhotski-Plemelj theorem this can be rewritten,

$$
\psi(\lambda ; \tau)=\lim _{\eta \rightarrow 0^{+}} \int_{-\infty}^{\infty} d m \frac{-1}{m+i \eta-\lambda} \tilde{J}(\lambda ; m) e^{-i m \tau}+2 \pi i \Theta(\operatorname{Im}\{\lambda\}) \tilde{J}(z ; \lambda) e^{-i \lambda \tau},
$$

where we understand the step-function to mean

$$
\Theta(x)= \begin{cases}1, & x>0 \\ 0, & x \leq 0\end{cases}
$$

$\psi(\lambda ; \tau)$ is clearly a solution of the inhomogeneous proper-time Dirac equation if both it and $\lambda \psi(\lambda ; \tau)$ exist and are in $C^{1}\left(\mathbb{R} ; \mathcal{H}_{\text {P.T. }}\right)$. We call it the retarded solution. To show why consider the case where $J(\tau)$ is supported only on $\tau>T_{1}$, $T_{1}<0$. Then we can use Eq. 98 to perform the $m$ integrals in Eq. $49 \mathrm{~b}$, looking at $\tau<T_{1}$. This gives

$$
\psi\left(\lambda ; \tau<T_{1}\right)=0 .
$$

The term "retarded solution" of course implies that this is the unique solution that satisfies this condition, which seems likely but which we do not have a proof for, due to the awkward properties of $\hat{H}$, and hence how little knowledge we have of its spectrum. This does not strictly affect our argument, though, since we do not use this condition as our definition.

We want to consider the response to a plane wave driving source. The solution, though, is not a well-defined section at $\lambda=-m_{0}$, and it cannot be directly defined as being zero before the source acts, since a plane-wave source acts forever. We therefore have to define it as a functional solution of the Dirac equation, reached as a limit of a sequence of $\mathcal{H}_{\text {P.T. }}$ solutions. Specifically, we say

Definition (retarded response to a plane wave) Say $J(\tau)=J e^{i m_{0} \tau}$. The retarded response to this source is given by the functional limit of the sequence of solutions given by Eq. 49a, 49b and 49c to the inhomogeneous proper-time Dirac equation with source profile 


$$
J_{\epsilon}(\tau)=A_{\epsilon}\left(m_{0} ; \tau\right) J:=e^{-\epsilon|\tau|+i m_{0} \tau} J
$$

as $\epsilon \rightarrow 0^{+}$.

$A_{\epsilon}\left(m_{0} ; \tau\right)$ is a nascent delta function in Fourier space, $\tilde{A}_{\epsilon}\left(m_{0} ; m\right) \rightarrow \delta\left(m_{0}+m\right)$ as $\epsilon \rightarrow 0^{+}$. Define $\psi_{\epsilon}(\lambda ; \tau)$ as the retarded solution of the inhomogeneous proper-time Dirac equation with source $A_{\epsilon}\left(m_{0} ; \tau\right) J$. Using Eq. $49 \mathrm{~b}$,

$$
\psi_{\epsilon}(\lambda ; \tau)=-\lim _{\eta \rightarrow 0^{+}} \int_{\mathbb{R}} d m \frac{\tilde{A}_{\epsilon}\left(m_{0} ; m\right) J(\lambda)}{m+i \eta-\lambda} e^{-i m \tau}+\mathcal{O}(\epsilon) .
$$

As $\epsilon \rightarrow 0^{+}$this does not have a well-defined value for $\lambda=-m_{0}$, but it does have a well-defined action as a functional. If $J(\lambda)$ and $\bar{\varphi}(\lambda)$ are bounded and continuous at $\lambda=-m_{0}$,

$$
\begin{aligned}
\langle\varphi, \psi(\tau)\rangle & =\lim _{\epsilon \rightarrow 0^{+}}\left(\varphi, \psi_{\epsilon}(\tau)\right)_{\mathrm{P} . \mathrm{T} .}=\lim _{\eta \rightarrow 0^{+}} \int_{\sigma(\hat{H})} d \mu_{\hat{H}}(\lambda) \frac{(\bar{\varphi}(\lambda), J(\lambda))_{\lambda}}{m_{0}+\lambda-i \eta} e^{i m_{0} \tau} \\
& =\left[P \int_{\sigma(\hat{H})} d \mu_{\hat{H}}(\lambda) \frac{(\bar{\varphi}(\lambda), J(\lambda))_{\lambda}}{m_{0}+\lambda}+i \pi\left\langle\phi, J\left(-m_{0}\right)\right\rangle\right] e^{i m_{0} \tau},
\end{aligned}
$$

where it is possible to bring the $\epsilon$ limit into the integral, once the $\eta$ limit has been brought outside. (Strictly, "continuous" should be interpreted as "Hölder-continuous" for the principal value to be well defined [24].) Therefore, the retarded response to a plane wave source $J(\tau) \in \mathcal{H}_{\hat{H}}$, of frequency $-m_{0}$, which is continuous at $\lambda=-m_{0}$, is a functional, $\psi(\tau)$, with a defined action on all $\varphi \in \mathcal{H}_{\hat{H}}$ for which $\bar{\varphi}$ is also continuous at $\lambda=-m_{0}$. This solution can also be seen as the limit of the $\mathcal{H}_{\text {P.T. }}$. states

$$
\psi_{\eta}(\lambda):=J(\lambda) /\left(m_{0}+\lambda-i \eta\right)
$$

as $\eta \rightarrow 0^{+}$. Using this,

$$
\left\langle\varphi,-\left(\hat{H}+m_{0}\right) \psi(\tau)\right\rangle=\lim _{\eta \rightarrow 0^{+}}\left(\varphi,-\left(\hat{H}+m_{0}\right) \psi_{\eta}\right)_{\mathrm{P} . \mathrm{T} .}=-(\varphi, J)_{\mathrm{P} . \mathrm{T} .} .
$$

Therefore $\psi(\tau)$, the retarded response to a plane wave of proper-time frequency $-m_{0}$, is a functional solution of the inhomogeneous spacetime Dirac equation (2) with mass $m_{0}$ and source profile $J$. This is the fundamental result that reflects Schwinger's operator relation (4).

\subsubsection{The Retarded Response to a Plane Wave Obeys the Feynman Boundary Condition}

The functional solution of the inhomogeneous proper-time Dirac equation projects onto a functional on some subset of $\mathcal{H}_{\text {S.T. }}$ at a particular time. By Eq. 33 this can be written, 


$$
\psi^{\text {in } / \text { out }}(\Pi ; t)=\lim _{\eta \rightarrow 0^{+}} \int_{\sigma(\hat{H})} d \mu_{\hat{H}}(\lambda) \frac{J^{\text {in } / \text { out }}(\Pi ; \lambda ; t)}{m_{0}+\lambda-i \eta}
$$

with $t \leq t_{\text {in }}$ or $t \geq t_{\text {out }}$. Use Eqs. 41c and $41 \mathrm{~d}$ to write

$$
\begin{aligned}
\psi_{\eta}^{\mathrm{in}}(\Pi ; t) & =\sum_{ \pm} \int_{\mathbb{R} \cup i I_{\mathrm{in}}^{+}} d \mu_{\hat{E}, \Pi}^{\mathrm{in}}(E) \frac{{ }_{ \pm} J(\Pi ; E) e^{-i E t}}{m_{0} \pm \lambda^{\mathrm{u}}(E, \Pi)-i \eta}, \quad t<t_{\text {in }} \\
\psi_{\eta}^{\text {out }}(\Pi, t) & =\sum_{ \pm} \int_{\mathbb{R} \cup i I_{\text {out }}^{-}} d \mu_{\hat{E}, \Pi}^{\text {out }}(E) \frac{{ }^{ \pm} J(\Pi ; E) e^{-i E t}}{m_{0} \pm \lambda^{1}(E, \Pi)-i \eta}, \quad t>t_{\text {out }},
\end{aligned}
$$

Define

$$
g_{\eta, \pm}^{\mathrm{u} / 1}(E, \Pi):=1 /\left(m_{0} \pm \lambda^{\mathrm{u} / \mathrm{l}}(E, \Pi)-i \eta\right),
$$

which is a meromorphic function in the upper/lower plane and uniformly bounded as $|E| \rightarrow \infty$. We can therefore use Eqs. $46 \mathrm{c}$ and $46 \mathrm{~d}$ with $g^{\mathrm{u} / 1}(E)=1$ to write

$$
\begin{aligned}
J^{\text {in }}(\Pi ; t) & =\sum_{ \pm} \int_{\mathbb{R}} d E_{ \pm} \breve{J}(\Pi ; E) \exp \left(-i E\left(t-t_{\text {in }}\right)\right) \\
J^{\text {out }}(\Pi, t) & =\sum_{ \pm} \int_{\mathbb{R}} d E^{ \pm} \breve{J}(\Pi ; E) \exp \left(-i E\left(t-t_{\text {out }}\right)\right)
\end{aligned}
$$

and with $g^{\mathrm{u} / \mathrm{l}}(E)$ as defined in Eq. 57,

$$
\begin{aligned}
\psi_{\eta}^{\text {in }}(\Pi ; t)= & \sum_{ \pm} \int_{\mathbb{R}} d E g_{\eta, \pm}^{\mathrm{u}}(E, \Pi)_{ \pm} \breve{J}(\Pi ; E) \exp \left(-i E\left(t-t_{\text {in }}\right)\right) \\
& -\int_{I_{\text {in }}^{+}} d \mu_{\hat{E}, \Pi}^{\text {in }}(p) R^{\mathrm{u}}\left(p, t-t_{\text {in }}\right)\left[g_{\eta, \pm}^{\mathrm{u}}\right]_{ \pm} J(\Pi ; p) \\
\psi_{\eta}^{\text {out }}(\Pi ; t)= & \sum_{ \pm} \int_{\mathbb{R}} d E g_{\eta, \pm}^{1}(E, \Pi)^{ \pm} \breve{J}(\Pi ; E) \exp \left(-i E\left(t-t_{\text {out }}\right)\right) \\
& -\int_{I_{\text {out }}^{-}} d \mu_{\hat{E}, \Pi}^{\text {out }}(p) R^{\mathrm{l}}\left(p, t-t_{\text {out }}\right)\left[g_{\eta, \pm}^{1}\right]^{ \pm} J(\Pi ; p) .
\end{aligned}
$$

We know that $J^{\text {in }}(\Pi, t)$ is supported only on $t>t_{\text {in }}$ and $J^{\text {out }}(\Pi, t)$ is supported only on $t<t_{\text {out }}$. In Appendix 3 we argue that we can treat both \pm components in the sum on the RHS of Eqs. 58a and 58b as also independently satisfying this condition. Therefore, we can use Eq. 98 to carry out the integrals, 


$$
\begin{aligned}
& \sum_{ \pm} \int_{\mathbb{R}} d E g_{\eta, \pm}^{\mathrm{u}}(E, \Pi)_{ \pm} \breve{J}(\Pi ; E) \exp \left(-i E\left(t-t_{\text {in }}\right)\right) \\
& \quad=2 \pi i \sum_{ \pm, E_{0} \in \text { poles }} \operatorname{Res}\left(g_{\eta, \pm}^{\mathrm{u}}\left(E_{0}, \Pi\right)\right)_{ \pm} \breve{J}\left(\Pi ; E_{0}\right) \exp \left(-i E_{0}\left(t-t_{\text {in }}\right)\right) \\
& \sum_{ \pm} \int_{\mathbb{R}} d E g_{\eta, \pm}^{1}(E, \Pi)^{ \pm} \breve{J}(\Pi ; E) \exp \left(-i E\left(t-t_{\text {out }}\right)\right) \\
& =-2 \pi i \sum_{ \pm, E_{0} \in \text { poles }} \operatorname{Res}\left(g_{\eta, \pm}^{1}\left(E_{0}, \Pi\right)\right) \pm \check{J}\left(\Pi ; E_{0}\right) \exp \left(-i E_{0}\left(t-t_{\text {out }}\right)\right)
\end{aligned}
$$

where the sum runs over poles in the upper and lower half plane in Eqs. 60a and $60 \mathrm{~b}$, respectively. This gives

$$
\begin{array}{r}
\psi_{\eta}^{\text {in }}(\Pi ; t)=2 \pi i \sum_{ \pm, E_{0}} \operatorname{Res}\left(g_{\eta, \pm}^{\mathrm{u}}\left(E_{0}, \Pi\right)\right) \rho\left(\Pi ; E_{0}\right) \\
{ }_{ \pm} J\left(\Pi ; E_{0}\right) \exp \left(-i E_{0}\left(t-t_{\text {in }}\right)\right) \\
\psi_{\eta}^{\text {out }}(\Pi ; t)=-2 \pi i \sum_{ \pm, E_{0}} \operatorname{Res}\left(g_{\eta, \pm}^{1}\left(E_{0}, \Pi\right)\right) \rho\left(\Pi ; E_{0}\right) \\
{ }_{ \pm} J\left(\Pi ; E_{0}\right) \exp \left(-i E_{0}\left(t-t_{\text {out }}\right)\right)
\end{array}
$$

Note that the integral over $p$ in Eq. 59 has cancelled with that in the definition of $\breve{J}$ in Eqs. $46 \mathrm{c}$ and $46 \mathrm{~d} . g_{\eta, \pm}^{\mathrm{u} / 1}(E, \Pi)$ has poles at

$$
E_{0}=\mp \sqrt{m_{0}^{2}+\Pi^{2}}+A_{0}^{\text {in/out }} \pm \frac{i \eta}{\sqrt{1+\left(\Pi^{2} / m_{0}^{2}\right)}}+\mathcal{O}\left(\eta^{2}\right)
$$

with residues $\pm m_{0} / \sqrt{m_{0}^{2}+\Pi^{2}}+\mathcal{O}(\eta)$. Therefore in the "in" case we capture the negative energy pole, and in the "out" case we capture the positive energy pole. Taking $\eta \rightarrow 0^{+}$

$$
\begin{gathered}
\psi^{\mathrm{in}}(\Pi ; t)=\frac{2 \pi i m_{0} \rho\left(\Pi ; E_{-}^{\mathrm{in}}\left(m_{0}, \Pi\right)\right)}{\sqrt{m_{0}^{2}+\Pi^{2}}}{ }_{-} J_{m_{0}}\left(\Pi ;-m_{0} ; t_{\mathrm{in}}\right) \\
\cdot \exp \left(-i\left[-\sqrt{m_{0}^{2}+\Pi^{2}}+e A_{0}^{\mathrm{in}}\right]\left(t-t_{\mathrm{in}}\right)\right)
\end{gathered}
$$




$$
\begin{gathered}
\psi^{\text {out }}(\Pi ; t)=\frac{2 \pi i m_{0} \rho\left(\Pi ; E_{+}^{\text {out }}\left(m_{0}, \Pi\right)\right)}{\sqrt{m_{0}^{2}+\Pi^{2}}}+J_{m_{0}}\left(\Pi ;-m_{0} ; t_{\text {out }}\right) \\
\cdot \exp \left(-i\left[\sqrt{m_{0}^{2}+\Pi^{2}}+e A_{0}^{\text {out }}\right]\left(t-t_{\text {out }}\right)\right),
\end{gathered}
$$

where we have used Eqs. 41a, 41b, 41c and 41d to write the RHS. It is clear that this is in $\mathcal{H}_{\text {S.T. }}$ as a direct consequence of our assumption that the generalised eigenvectors projected onto times $t \leq t_{\text {in }}$ and $t \geq t_{\text {out }}$ take values in $\mathcal{H}_{\text {S.T. }}$ (30). It is also clear from Eq. 22 that it obeys the Feynman boundary condition.

\section{The "Causal Propagator" of Fradkin, Gitman \& Shvartsman}

FGS [16] require that their external field's instantaneous energy spectrum at $t_{\text {in }}$ and $t_{\text {out }}$ has a gap that covers $E=0$. They also assume that the Hamiltonian at $t_{\text {in }}$ and $t_{\text {out }}$, $\hat{\mathscr{H}}_{m_{0}}^{\text {jnout }}$, has a complete eigenbases in $\mathcal{H}_{\mathrm{S} \text {.T. }}$. They define two decompositions of the Schrödinger-picture field operator,

$$
\begin{gathered}
\hat{\psi}_{S}(\mathbf{x})=\sum_{\alpha}{ }_{+} \varphi_{\alpha}(\mathbf{x}) \hat{a}_{\alpha}\left(t_{\mathrm{in}}\right)+{ }_{-} \varphi_{\alpha}(\mathbf{x}) \hat{b}_{\alpha}^{\dagger}\left(t_{\mathrm{in}}\right) \\
=\sum_{\alpha}{ }^{+} \varphi_{\alpha}(\mathbf{x}) \hat{a}_{\alpha}\left(t_{\mathrm{out}}\right)+{ }^{-} \varphi_{\alpha}(\mathbf{x}) \hat{b}_{\alpha}^{\dagger}\left(t_{\mathrm{out}}\right) .
\end{gathered}
$$

$\left\{{ }_{+}-\varphi_{\alpha}(\mathbf{x})\right\}_{\alpha}$ are the instantaneous eigenstates $\hat{\mathscr{H}}_{m_{0}}$ with positive/negative energy, and $\left\{{ }^{+/-} \varphi_{\alpha}(\mathbf{x})\right\}_{\alpha}$ are the instantaneous eigenstates $\hat{\mathscr{H}}_{m_{0}}^{\text {bt }}$ with positive/negative energy. These in turn define two bipartitions of the Hilbert space $\mathcal{H}_{\text {S.T. }}$ into the two spanned by the positive and the negative energy eigenstates: ${ }_{ \pm} \varphi_{\alpha}$ spans ${ }_{ \pm} \mathcal{H}_{\text {S.T. }}^{m_{0}}$ and ${ }^{ \pm} \varphi_{\alpha}$ spans ${ }^{ \pm} \mathcal{H}_{\text {S.T. }}^{m_{0}}$ as defined above Eq. 21. These bases define the creation/annihilation operators used in the decomposition, which FGS in turn use to define in/out vacuum states,

$$
\hat{a}_{\alpha}\left(t_{\text {in }}\right)\left|0, t_{\text {in }}\right\rangle=\hat{b}_{\alpha}\left(t_{\text {in }}\right)\left|0, t_{\text {in }}\right\rangle=\hat{a}_{\alpha}\left(t_{\text {out }}\right)\left|0, t_{\text {out }}\right\rangle=\hat{b}_{\alpha}\left(t_{\text {out }}\right)\left|0, t_{\text {out }}\right\rangle=0 .
$$

Note that the vacua are invariant under changes of basis that leave ${ }_{ \pm} \mathcal{H}_{\text {S.T. }}^{m_{0}}$ and $\pm \mathcal{H}_{\text {S.T. }}^{m_{0}}$ invariant. Therefore, the choice of vacuum is in one-to-one correspondence with the possible bipartitions of $\mathcal{H}_{\text {S.T. }}$ into two orthogonal subspaces. FGS define the "causal propagator", with $t_{\text {out }} \geq x^{0}, y^{0} \geq t_{\text {in }}$, by the expression

$$
\left.S^{c}(x, y):=i\left\langle 0, \text { out }\left|T\left\{\hat{\psi}_{H}(x) \hat{\bar{\psi}}_{H}(y)\right\}\right| 0, \text { in }\right\rangle c_{v}^{-1}, \quad c_{v}:=\langle 0, \text { out }| 0, \text { in, }\right\rangle
$$

where $\mid 0$, in $\rangle, \mid 0$, out $\rangle$ and $\hat{\psi}_{H}(x)$ are $\left|0, t_{\text {in }}\right\rangle,\left|0, t_{\text {out }}\right\rangle$ and $\hat{\psi}_{S}$ in the Heisenberg picture, respectively. Define the "causal" solution of the inhomogeneous spacetime Dirac equation, 


$$
\psi^{c}(x):=\int d^{4} x^{\prime} S^{c}\left(x, x^{\prime}\right) J\left(x^{\prime}\right)
$$

Define the components of an element $f \in \mathcal{H}_{\text {S.T. }}$. with respect to the in and out bases, $\left\{{ }_{ \pm} f_{\alpha}\right\}_{\alpha}$ and $\left\{{ }^{ \pm} f_{\alpha}\right\}_{\alpha}$, as

$$
f(\mathbf{x})=\sum_{\alpha}\left[{ }_{+} f_{\alpha+} \varphi_{\alpha}(\mathbf{x})+{ }_{-} f_{\alpha-} \varphi_{\alpha}(\mathbf{x})\right]=\sum_{\alpha}\left[{ }^{+} f_{\alpha}{ }^{+} \varphi_{\alpha}(\mathbf{x})+{ }^{-} f_{\alpha}{ }^{-} \varphi_{\alpha}(\mathbf{x})\right] .
$$

We can take the $\mathcal{H}_{\text {S.T. }}$ product of the field operator (64) with a $\mathbb{C}$-valued field, which returns an operator,

$$
\begin{aligned}
\left(\hat{\psi}_{S}, f\right)_{\text {S.T. }} & =\int d^{3} \mathbf{x} \hat{\psi}_{S}^{\dagger}(\mathbf{x}) f(\mathbf{x})=\sum_{\alpha}\left({ }_{+} f_{\alpha} \hat{a}_{\alpha}^{\dagger}\left(t_{\text {in }}\right)+{ }_{-} f_{\alpha} \hat{b}_{\alpha}\left(t_{\text {in }}\right)\right) \\
& =\sum_{\alpha}\left({ }^{+} f_{\alpha} \hat{a}_{\alpha}^{\dagger}\left(t_{\text {out }}\right)+{ }^{-} f_{\alpha} \hat{b}_{\alpha}\left(t_{\text {out }}\right)\right) .
\end{aligned}
$$

Note $\left[\left(f, \hat{\psi}_{S}\right)_{\text {S.T. }}\right]^{\dagger}=\left(\hat{\psi}_{S}, f\right)_{\text {S.T. }}$. This, with Eq. 65 , gives the conditions

$$
\begin{gathered}
\left\langle 0, t_{\text {in }}\left|\left(\hat{\psi}_{S}, f\right)_{\text {S.T. }}=\left(f, \hat{\psi}_{S}\right)_{\text {S.T. }}\right| 0, t_{\text {in }}\right\rangle=0 \quad \text { if } \quad f \in{ }_{+} \mathcal{H}_{\text {S.T. }}^{m_{0}} \\
\left(\hat{\psi}_{S}, f\right)_{\text {S.T. }}\left|0, t_{\text {out }}\right\rangle=\left\langle 0, t_{\text {out }}\right|\left(f, \hat{\psi}_{S}\right)_{\text {S.T. }}=0 \quad \text { if } \quad f \in{ }^{-} \mathcal{H}_{\text {S.T. }}^{m_{0}} .
\end{gathered}
$$

Take the Hilbert-space product of some $f \in \mathcal{H}_{\text {S.T. }}$ with the causal solution at $t_{\text {in }}$,

$$
\begin{aligned}
\left(f, \psi^{c}\left(t_{\text {in }}\right)\right)_{\text {S.T. }} & =-i \sum_{l} \int d^{4} y J_{l}(y)\left\langle 0, \text { out }\left|\hat{\bar{\psi}}_{H \mid l}(y)\left[\left(f, \hat{\psi}_{H}\left(t_{\text {in }}\right)\right)_{\text {S.T. }}\right]\right| 0, \text { in }\right\rangle \\
& =-i \sum_{l} \int d^{4} y J_{l}(y)\left\langle 0, \text { out }\left|\hat{\bar{\psi}}_{H \mid l}(y) \hat{U}\left(0, t_{\text {in }}\right)\left[\left(f, \hat{\psi}_{S}\right)_{\text {S.T. }}\right]\right| 0, t_{\text {in }}\right\rangle \\
& =0 \quad \text { if } f \in{ }_{+} \mathcal{H}_{\text {S.T. }}^{m_{0}} .
\end{aligned}
$$

and by an analagous calculation at $t_{\text {out }}$,

$$
\left(f, \psi^{c}\left(t_{\text {out }}\right)\right)=0 \quad \text { if } \quad f \in{ }^{-} \mathcal{H}_{\text {S.T. }}^{m_{0}}
$$

Due to the orthogonality of the subspaces for real $m_{0}$, these are equivalent to the Feynman boundary condition.

\section{Three Other Equivalent Solution Forms}

\subsection{Schwinger's Proper-Time Propagator}

Use Eq. 53 and 


$$
\frac{1}{x-i \eta}=i \int_{0}^{\infty} d s e^{-i(x-i \eta) s}, \quad x \in \mathbb{R}, \eta>0
$$

to get

$$
\begin{aligned}
\psi_{\eta}(\lambda) & =i \int_{0}^{\infty} d s e^{-i\left(m_{0}-i \eta\right) s} e^{-i \lambda s} J(\lambda) \\
& =\left[\left(i \int_{0}^{\infty} d s e^{-i\left(m_{0}-i \eta\right) s} e^{-i \hat{H} s}\right) J\right](\lambda) .
\end{aligned}
$$

The term in round brackets is Schwinger's expression for his Green's function (4), with an infinitesimal negative imaginary inserted. Schwinger inserted the infinitesimal negative imaginary during calculations that would otherwise explicitly return a divergence. Later authors [5] formalised this procedure, and say that the expression is defined for negative imaginary mass, with the prescription that solutions for the real mass are to be derived by then taking the limit of the imaginary component to zero, perhaps after appropriate integrations. In this work this procedure is understood as returning a functional solution of the Dirac equation with a domain smaller than $\mathcal{H}_{\text {P.T. }}$, with action $\langle\phi, \psi\rangle=\lim _{\eta \rightarrow 0^{+}}\left(\phi, \psi_{\eta}\right)_{\text {P.T. }}$.

\subsection{The $m^{2}-i \epsilon$ Prescription and Continuation from Euclidean Time}

Consider a solution of the inhomogeneous spacetime Dirac equation with mass $m_{0}$ that takes values in $\mathcal{H}_{\text {S.T. }}$ for $t \leq t_{\text {in }}$ and $t \geq t_{\text {out }}$, before and after the source acts. At these times they are just solutions to the homogeneous spacetime Dirac equation, so we can write, in the $\mathcal{H}_{\text {in/out },|\hat{\Pi}|}$ representations according to Eqs. 20a and 20b,

$$
\begin{gathered}
\psi^{\text {in }}(\Pi ; t)=\sum_{ \pm}{ }_{ \pm} \psi_{m_{0}}\left(\Pi ; t^{\prime}\right) \exp \left(-i\left[ \pm \sqrt{m_{0}^{2}+\Pi^{2}}+e A_{0}^{\text {in }}\right]\left(t-t^{\prime}\right)\right) \\
\psi^{\text {out }}(\Pi ; t)=\sum_{ \pm}{ }^{ \pm} \psi_{m_{0}}\left(\Pi ; t^{\prime}\right) \exp \left(-i\left[ \pm \sqrt{m_{0}^{2}+\Pi^{2}}+e A_{0}^{\text {out }}\right]\left(t-t^{\prime}\right)\right) .
\end{gathered}
$$

Now consider that we demand that these solutions are each analytic functions of $m_{0}$ in some open region, confined to the lower half complex plane, which has $m_{0}$ as a limit point. Further, we demand that in this region, no component grows exponentially. The $m_{0}$ dependence in the ${ }_{ \pm} \psi_{m_{0}}\left(\Pi ; t^{\prime}\right)$ and ${ }^{ \pm} \psi_{m_{0}}\left(\Pi ; t^{\prime}\right)$ terms are here insignificant, so we can focus on the mass-dependence in the exponential, which is in fact a dependence on $m_{0}^{2}$. Specifically, we can enforce this demand by asking that there exists an $\epsilon>0$ such that for all masses $m^{2}(\delta):=m_{0}^{2}-i \delta$, for all $\delta \in(0, \epsilon]$, the expression (75) does not grow exponentially as $t \rightarrow \pm \infty$. This is true if and only if $+\psi_{m_{0}}\left(\Pi ; t \leq t_{\text {in }}\right)={ }^{-} \psi_{m_{0}}\left(\Pi ; t \geq t_{\text {out }}\right)=0$, which is the Feynman boundary condition.

Next, consider making the same demand, that the solution does not grow exponentially, if we enact the replacement 


$$
t \rightarrow e^{-i \phi} t, \quad \phi \in(0, \pi / 2]
$$

So long as $\left|e A_{0}^{\text {in/out }}\right|<m_{0}$, this enforces exactly the same requirement. This is a version of the idea that the causal solutions are to be derived by analytic continuation of solutions for "Euclidean time" [25]. Both these requirements are therefore equivalent to imposing the Feynman boundary condition. Note that this demand for a lack of exponential growth is often enforced in the literature implicitly. One way of doing so is to demand that the solution has a Fourier transform. This is the most common way in less mathematically rigorous works that use the " $m^{2}-i \epsilon$ prescription". Another way of doing so is to demand that the solutions are tempered distributions. This is the procedure used in axiomatic treatments of Euclidean green's functions [25].

There is cause for caution here. To get the same boundary condition, we have needed to demand that the actual solutions for imaginary mass or imaginary time obey certain constraints on growth at infinity, not the terms of a perturbative series. If we were to demand that each of the solutions that form the asymptotic series approximation to the correct solution do not exponentially grow at infinity, then this would be neither necessary nor sufficient for their sum to not exponentially grow at infinity. This explains Gitman's observation [12] that FGS's causal propagator does not equal that derived by applying the $m^{2}-i \epsilon$ prescription to the perturbative series expression of the propagator. In Feynman-diagrammatic terms, the important thing for the $m^{2}-i \epsilon$ or Euclidean-time prescriptions to give the Feynman boundary condition is that the "leg" connecting the input and output particles to infinity is treated as propagation under the external field, not as a free propagator.

\section{Discussion}

\subsection{The Result}

The principal result of this paper is the demonstration that, for a broad class of external fields (those satisfying the form (3)) and under reasonable mathematical assumptions, Schwinger's proper-time propagator, FGS's causal propagator, and propagators derived by analytic continuation of the complexified mass or time all obey what we call the "Feynman boundary condition". This can loosely be stated as the requirement that they propagate positive-energy solutions into the future and negative-energy solutions into the past. Under a further assumption discussed in Sect. 2.2.3, that there exists no solution to the homogeneous equation which is wholly negative-energy at the initial time and wholly positive-energy at the final time, this is a demonstration of the equivalence of all these propagators. To derive this result, we have interpreted Schwinger's operator relationship (4) as a statement relating the solutions of two partial differential equations: the retarded solution of the inhomogeneous proper-time Dirac equation (11) for a plane-wave driving source of proper-time frequency $-m_{0}$ is a solution of the inhomogeneous spacetime Dirac equation (2) with mass $m_{0}$. When applied to fields of form (3), this also gives a novel expression for this solution before the in-time and after the out-time in terms 
of the decomposition of the source profile in the generalised eigenbasis of $\hat{H}$ (63). This result might be of independent interest. We now discuss the two caveats just mentioned, the class of external field and the mathematical assumptions made, in turn.

\subsection{Conditions on the External Field}

While Schwinger's propagator and the proper-time method described in Sect. 2 have been demonstrated to be wholly equivalent for any external field, the other three propagators considered have only been demonstrated as equivalent by their obedience of the Feynman boundary condition. Their obedience of the Feynman boundary condition is contingent on the field satisfying the conditions (3), so equivalence has only been demonstrated for fields satisfying these conditions. There are also some subtle distinctions in the conditions applied to some of the propagators, which we now discuss.

First, the continuation from Euclidean time and FGS's causal propagator require $\left|e A_{0}^{\text {in/out }}\right|<m_{0}$, such that the "negative energy" states genuinely all have negative energy and the "positive energy" states all have positive energy. The proper-time method and the $m^{2}-i \epsilon$ prescription do not. This is a technicality entangled with issues to do with the normalisation with respect to the vacuum, and besides can be fixed by a partial gauge constraint.

More substantially, FGS's causal propagator is only defined for times $t_{\text {out }} \geq t \geq t_{\text {in }}$. Say the field used for FGS's causal propagator is $A_{\mu}(x)$, and define

$$
A_{\mu}^{\prime}\left(t, \mathbf{x} ; t_{\text {in }}, t_{\text {out }}\right):=\left\{\begin{array}{l}
A_{\mu}\left(t_{\text {out }}, \mathbf{x}\right), \quad t \geq t_{\text {out }} \\
A_{\mu}(t, \mathbf{x}), \quad t_{\text {out }} \geq t \geq t_{\text {in }} \\
A_{\mu}\left(t_{\text {in }}, \mathbf{x}\right), \quad t \leq t_{\text {in }} .
\end{array}\right.
$$

$A_{\mu}^{\prime}$ satisfies the conditions (3) if and only if $A_{\mu}(x)$ has no spatial variation at $t_{\text {in }}$ or $t_{\text {out }}$. Therefore, the solution derived using the proper-time method, the $m^{2}-i \epsilon$ prescription and continuation from Euclidean time matches on this temporal region if we use the field $A_{\mu}^{\prime}\left(x ; t_{\text {in }}, t_{\text {out }}\right)$ and $A_{\mu}(x)$ has no spatial variation at $t_{\text {in }}$ or $t_{\text {out }}$. We can therefore, using Eq. 77, derive a solution using any of the former three methods that matches that derived using FGS's causal propagator on the region $t_{\text {out }} \geq t \geq t_{\text {in }}$ with a field constrained only by a partial gauge condition.

FGS derive a solution for all time by taking the limits $t_{\text {in }} \rightarrow-\infty, t_{\text {out }} \rightarrow \infty$. We therefore have

$$
\lim _{t_{\text {in }} \rightarrow-\infty, t_{\text {out }} \rightarrow \infty} \psi\left[A_{\mu}^{\prime}\left(t_{\text {in }}, t_{\text {out }}\right)\right](x)=\lim _{t_{\text {in }} \rightarrow-\infty, t_{\text {out }} \rightarrow \infty} \psi \psi_{\mathrm{FGS}}^{t_{\text {in }}, t_{\text {out }}}\left[A_{\mu}\right](x),
$$

where $\psi_{\mathrm{FGS}}^{t_{\mathrm{in}}, t_{\text {out }}}\left[A_{\mu}\right]$ returns the solutions to the inhomogeneous equation with the external field $A_{\mu}(x)$ derived using FGS's causal propagator with the given in and out times, and $\psi\left[A_{\mu}\right]$ returns the solution derived by any other the other three methods. If $A_{\mu}(x)$ satisfies conditions ( 3 ) for any finite $t_{\text {in }}$ and $t_{\text {out }}$, then $A_{\mu}^{\prime}\left(x ; t_{\text {in }} ; t_{\text {out }}\right)=A_{\mu}(x)$ 
for sufficiently large-magnitude $t_{\text {in }}$ and $t_{\text {out }}$, and hence we get equality of all four methods on all time,

$$
\psi\left[A_{\mu}\right](x)=\lim _{t_{\mathrm{in}} \rightarrow-\infty, t_{\mathrm{out}} \rightarrow \infty} \psi_{\mathrm{FGS}}^{t_{\mathrm{in}}, t_{\mathrm{out}}}\left[A_{\mu}\right](x) .
$$

We have therefore demonstrated the equality of all four propagators for all fields that obey the constraints (3) for all time. In this sense, equality has been proven for all "physical" situations, in which we can confine the action of the external field to some finite time. The limitation is important to state, though, since fields that do not obey condition (3) include such important theoretical cases as the everywhere uniform electric field considered by Schwinger [4]. FGS prove equivalence in this specific case by direct calculation [16], though, so there is some reason to suspect that the proper time and FGS propagators are equivalent in all cases. This paper's result could be extended to cover this general case if we knew that the mapping from external field profiles to inhomogeneous spacetime Dirac equation solutions, with fixed source profile, was continuous, and hence

$$
\lim _{t_{\text {in }} \rightarrow-\infty, t_{\text {out }} \rightarrow \infty} \psi\left[A_{\mu}^{\prime}\left(t_{\text {in }}, t_{\text {out }}\right)\right](x)=\psi\left[A_{\mu}(x)\right] .
$$

\subsection{Mathematical Assumptions}

\subsubsection{The Green's Function is Well-Defined as a Mapping from Compactly Supported Source Profiles}

We assume that the ascription of a (functional) solution of the inhomogeneous equation for every source profile that is compactly supported in time defines the Green's function well. There is no single mathematical result that justifies this, since we are demonstrating the equivalence of Green's functions as used in literature which does not share a strict definition of what mathematical object the Green's function is. Schwinger's definition of $\hat{G}$ as an operator on the Hilbert space is insufficient, as even when looking at our restricted class of source profiles, the solutions returned are not necessarily in the Hilbert space. To give the idea plausibility, consider that in axiomatic treatments of QFT [25] the Green's functions are often treated as tempered distributions on $\mathbb{R}^{3 \oplus 1} \otimes \mathbb{R}^{3 \oplus 1}$. In this case, we can use the Schwartz kernel theorem to prove an isomorphism between this class and continuous linear mappings from source profiles in the Schwartz class to tempered-distributional solutions of the inhomogeneous equation [26]. The continuity of this mapping ensures that defining it on the compactly supported functions defines it well on the Schwartz class, since the compactly supported functions are dense in the Schwartz class. 


\subsubsection{Neglecting the $m= \pm i \Pi$ Case in Sect. 2.2}

All uses we make of our $\mathcal{H}_{\text {S.T. }}$ representations in which $\hat{\mathscr{H}}_{m}^{\text {in/out }}$ for imaginary $m$ is diagonal proceeds from Eq. 33. Suppose in this equation, we set $\phi^{\text {in/out }}(\Pi ; \lambda ; t)=0$ for $\lambda= \pm i \Pi$. This would only alter the LHS of the equation if $i \Pi$ or $-i \Pi$ were in the point spectrum of $\hat{H}$. Assuming this to be the case, for this alteration of the LHS to affect the Hilbert space state that $\phi^{\text {in/out }}(\Pi ; t)$ represents, we require $\phi^{\text {in/out }}(\Pi ; t)$ to be altered on a region of finite $\mu_{\text {in/out }}$-measure. Since the point spectrum of $\hat{H}$ covers a region of Lebesgue-measure 0 , the only way for this to be the case would be if $\Pi$ were in the point spectrum of $\hat{\Pi}^{\text {in/out }}$. Therefore, the neglect of the case where $m= \pm i \Pi$ can only matter if there exists a real number $\alpha$ which is in the point spectrum of $\hat{\Pi}^{\text {in/out }}$, and $\pm i \alpha$ is in the point spectrum of $\hat{H}$. We therefore must assume this not to be the case.

\subsubsection{Other Assumptions}

There are three further classes of substantial mathematical assumptions made in this paper. The first class is that two operators which are known to by symmetric, $\hat{\Pi}$ and $\hat{H}^{2}$, are self-adjoint, as mentioned in Sect. 2.1. Proving that Hamiltonians with various potentials are self-adjoint is a known, difficult problem in the case of non-relativistic quantum mechanics [27]. The extension of this work to the proper-time case would be substantial. The second class is that the relevant solutions to the homogeneous and inhomogeneous spacetime Dirac equation are equal to $\mathcal{H}_{\text {S.T. }}$ states at times $t \leq t_{\text {in }}$ and $t \geq t_{\text {out }}$ (30), and that they do not grow exponentially in the future or past. The third class is that we can exchange the order of various infinite sums and integrals, as done to derive Eqs. 28, 33, 34 and 46c,d.

These are all the sorts of assumptions that are frequently made implicitly in calculations performed in the bra-ket formalism, where, respectively, unbounded operators are treated as operating on the whole Hilbert space, their eigenvectors are treated as if in the Hilbert space, and integrals and infinite summations over ket-vector bases are manipulated symbolically as finite summations. A significant reason it has been necessary to make them is that we have used very little knowledge of $\hat{H}$ : in particular its spectrum and the properties of its generalised eigenstates are largely unknown. A substantial work of functional analysis of $\hat{H}$ as an operator on $\mathcal{H}_{\text {P.T. }}$ would be needed to validate our assumptions, which would probably necessitate some regularity constraints on the external field $A_{\mu}(x)$. This would act as an extension of some of the results derived from functional analysis for putting regular quantum mechanics on a mathematically rigorous footing $[21,28]$ to Schwinger's proper-time quantum mechanics. This paper has not supplied the proofs needed for this extension. We believe it is clear, though, that the assumptions made are no more egregious than are commonplace in physics literature that uses the bra-ket formalism, and in making its assumptions explicit acts as a derivation of some plausible sufficient conditions for the validity of the proper-time method. The validation of these assumptions, or the derivation of fewer or less restrictive assumptions, is a substantial area for future work. 


\section{Appendix 1: Complex Fourier Analysis}

The methods in this subsection are guided closely by [29, Chap. 19]. Following its notation, we label the real-variable we are transforming in this section $t$, the real Fourier variable $x$, and the complex variable $z=x+i y$. We can write the complex Fourier transform as

$$
f(z)=\int_{\mathbb{R}} d t e^{i z t} F(t) .
$$

We write a real Fourier transform and its inverse as

$$
\mathcal{F}_{x, t}[G(t)]=\int_{-\infty}^{\infty} d t e^{i x t} G(t), \quad \mathcal{F}_{t, x}^{-1}[g(x)]=\frac{1}{2 \pi} \int_{-\infty}^{\infty} d x e^{-i x t} g(x) .
$$

Suppose $F^{\mathrm{l}}(t)$ is supported only on $(-\infty, A)$, and $F^{\mathrm{u}}(t)$ only on $(B, \infty)$, with $A>0$, $B<0 . f^{\mathrm{u} / \mathrm{l}}(z)$ is the complex Fourier transform of these functions. Both functions are square-integrable, and by the Plancherel theorem,

$$
\int_{-\infty}^{\infty} d t\left|F^{\mathrm{u} / 1}(t)\right|^{2}=\int_{\mathbb{R}} d x\left|f^{\mathrm{u} / 1}(x)\right|^{2}=C>0 .
$$

By the Paley-Wiener theorems, we know that $f^{\mathrm{u} / 1}(z)$ is analytic on the upper/lower half complex plane. Now, define the inverse Fourier transform along some horizontal line defined by $y$ constant as

$$
\tilde{f}_{y}(t)=\frac{1}{2 \pi} \int_{-\infty}^{\infty} d x e^{-i x t} f(x+i y)=\mathcal{F}_{t, x}^{-1}[f(x+i y)]
$$

and write

$$
f(x+i y)=\int_{-\infty}^{\infty} d t e^{i(x+i y) t} F(t)=\mathcal{F}_{x, t}\left[e^{-y t} F(t)\right]
$$

to give

$$
\tilde{f}_{y}(t)=\mathcal{F}_{t, x}^{-1}\left[\mathcal{F}_{x, t}\left[e^{-y t} F(t)\right]\right]=e^{-y t} F(t)
$$

Now consider

$$
\begin{aligned}
\int_{-\infty}^{\infty} d x\left|f^{\mathrm{u} / 1}(x+i y)\right|^{2} & =\int_{-\infty}^{\infty} d t\left|\mathcal{F}_{t, x}^{-1}\left[f^{\mathrm{u} / 1}(x+i y)\right]\right|^{2} \\
& =\int_{-\infty}^{\infty} d t\left|\tilde{f}_{y}^{\mathrm{u} / 1}(t)\right|^{2}=\int_{-\infty}^{\infty} d t e^{-2 t y}\left|F^{\mathrm{u} / 1}(t)\right|^{2} \\
& \leq \begin{cases}e^{-2 A y} C, & \text { l case, } \quad y<0 \\
e^{-2 B y} C, & \text { u case }, \quad y>0,\end{cases}
\end{aligned}
$$


where in the first line we have used the Plancherel theorem, in the second we have used Eq. 86 and in the third we have used the known support of $F^{\mathrm{u} / 1}(t)$ to extract a maximum value of $e^{-2 t y}$ and used Eq. 83. Now write

$$
\begin{aligned}
\int_{-\infty}^{\infty} d x\left|f^{\mathrm{u} / 1}(x+i y) e^{-i t(x+i y)}\right|^{2} & =e^{2 y t} \int_{-\infty}^{\infty} d x\left|f^{\mathrm{u} / 1}(x+i y)\right|^{2} \\
& \leq\left\{\begin{array}{lll}
e^{2(t-A) y} C, & 1 \text { case }, & y<0 \\
e^{2(t-B) y} C, & \text { u case }, & y>0
\end{array}\right.
\end{aligned}
$$

Next, write the interval $I_{y}^{\mathrm{u}}=[y, 0]$, with $y<0$ assumed, and $I_{y}^{1}=[0, y]$, with $y>0$ assumed. Then define the positive integral

$$
\Lambda^{\mathrm{u} / \mathrm{l}}(\alpha ; y):=\int_{I_{y}^{\mathrm{u} / 1}} d u\left|f^{\mathrm{u} / 1}(\alpha+i u) e^{-i t(\alpha+i u)}\right|^{2} .
$$

Now consider

$$
\int_{-\infty}^{\infty} d \alpha \Lambda^{\mathrm{u} / \mathrm{l}}(\alpha ; y)=\int_{I_{y}^{\mathrm{u} / 1}} d u\left(\int_{-\infty}^{\infty} d \alpha\left|f^{\mathrm{u} / \mathrm{l}}(\alpha+i u) e^{-i t(\alpha+i u)}\right|^{2}\right),
$$

where Fubini's theorem allows us to change the order of the integral. We can now use Eq. 88 to get

$$
\begin{aligned}
& \int_{-\infty}^{\infty} d \alpha \Lambda^{\mathrm{l}}(\alpha ; y) \leq C \int_{I_{y}^{1}} d u e^{2(t-A) u}=\frac{C}{2(t-A)}\left(1-2 e^{2(t-A) y}\right) \\
& \int_{-\infty}^{\infty} d \alpha \Lambda^{\mathrm{u}}(\alpha ; y) \leq C \int_{I_{y}^{\mathrm{u}}} d u e^{2(t-B) u}=\frac{C}{2(t-B)}\left(1-e^{2(t-B) y}\right) .
\end{aligned}
$$

Therefore for every fixed $y, \Lambda^{\mathrm{u} / 1}(\alpha, y)$ is an integrable function of $\alpha$ on the real line. This means that there exists a sequence, $\left\{\gamma_{j} \in \mathbb{R}\right\}_{j}$, such that as $j \rightarrow \infty$ [30],

$$
\gamma_{j} \rightarrow+\infty, \quad \Lambda^{\mathrm{u} / 1}\left(\gamma_{j}, y\right)=0 .
$$

Define $\Gamma_{\alpha, \beta}^{\mathrm{u}}$ as the anticlockwise rectangular contour with corners at $( \pm \alpha, 0),( \pm \alpha, \beta)$, $\alpha, \beta \in \mathbb{R}^{+}$, which does not include the real line. Define, similarly, $\Gamma_{\alpha, \beta}^{l}$ as the clockwise contour with corners at $( \pm \alpha, 0),( \pm \alpha,-\beta)$ which does not include the real line. Also suppose the function $h^{\mathrm{u} / 1}(z)$ has bounded absolute value $\left|h^{\mathrm{u} / 1}(z)\right|$ as $|z| \rightarrow \infty$ on the upper/lower complex plane, and is meromorphic on the upper/lower complex plane. Then we can write

$$
\begin{aligned}
& \int_{\Gamma_{\gamma_{j}, \beta}^{\mathrm{l}}}|d z|\left|h^{1}(z) f^{1}(z) e^{-i z t}\right|^{2} \leq \operatorname{Sup}_{\Gamma_{\gamma_{j}, \beta}^{\mathrm{l}}}\left(\left|h^{1}(z)\right|^{2}\right)\left(\int_{-\gamma_{j}}^{\gamma_{j}} d x\left|f^{1}(x-i \beta) e^{-i t(x-i \beta)}\right|^{2}\right. \\
& \left.\quad+\Lambda^{1}\left(\gamma_{j},-\beta\right)+\Lambda^{1}\left(-\gamma_{j},-\beta\right)\right)
\end{aligned}
$$




$$
\begin{aligned}
& \int_{\Gamma_{\gamma_{j}, \beta}^{\mathrm{u}}}|d z|\left|h^{\mathrm{u}}(z) f^{\mathrm{u}}(z) e^{-i z t}\right|^{2} \leq \operatorname{Sup}_{\Gamma_{\gamma_{j}, \beta}^{\mathrm{u}}}\left(\left|h^{\mathrm{u}}(z)\right|^{2}\right)\left(\int_{-\gamma_{j}}^{\gamma_{j}} d x\left|f^{\mathrm{u}}(x+i \beta) e^{-i t(x+i \beta)}\right|^{2}\right. \\
& \left.\quad+\Lambda^{\mathrm{u}}\left(\gamma_{j}, \beta\right)+\Lambda^{\mathrm{u}}\left(-\gamma_{j}, \beta\right)\right) .
\end{aligned}
$$

If we take $j \rightarrow \infty$, then the second two terms in the brackets vanishes by Eq. 93. If we then take $\beta \rightarrow \infty$ then the first term vanishes by Eq. 88, so long as $t<B$ in the "u" case and $t>A$ in the "l" case.. $\operatorname{Sup}_{\Gamma_{\gamma_{j}, \beta}^{\mathrm{u} / 1}}\left(\left|h^{\mathrm{u} / 1}(z)\right|^{2}\right)$ stays finite by assumption, and hence

$$
\lim _{\beta \rightarrow \infty} \lim _{j \rightarrow \infty} \int_{\Gamma_{\gamma_{j}, \beta}^{\mathrm{u} / 1}}|d z|\left|h^{\mathrm{u} / 1}(z) f^{\mathrm{u} / 1}(z) e^{-i z t}\right|^{2}=0 .
$$

Therefore, there exists a way of taking $\Gamma^{\mathrm{u} / 1}$ to be an infinite rectangular contour in the upper/lower half plane excluding the real line, such that

$$
\int_{\Gamma^{\mathrm{u} / 1}} d z h^{\mathrm{u} / \mathrm{l}}(z) f^{\mathrm{u} / \mathrm{l}}(z) e^{-i z t}=0,
$$

and hence, by the residue theorem,

$$
\int_{\mathbb{R}} d z h^{\mathrm{u} / 1}(z) f^{\mathrm{u} / 1}(z) e^{-i z t}= \pm 2 \pi i \sum_{z_{0} \in \text { poles }} f\left(z_{0}\right) \operatorname{Res}\left(h^{\mathrm{u} / 1}\left(z_{0}\right)\right),
$$

if $t<B$ in the "u" case and if $t>A$ in the case "l" case. $\pm=+/-$ in the "u/l" case, and the $z_{0}$ sum runs over poles in the upper/lower half plane.

\section{Appendix 2: The Time-Translation Operator of the Dirac Equation}

We here prove that the classical initial value problem for the Dirac equation with complex mass is well-posed: any initial value gives one and only one classical solution. To do this, we write the Dirac equation in the form

$$
\begin{gathered}
\partial_{t} \psi=(\hat{A}+\hat{B}) \psi \\
\hat{A}:=-\gamma^{0} \hat{\Pi}^{\text {in/out }}-i A_{0}^{\text {in/out }}, \quad \hat{B}:=-i m \gamma^{0},
\end{gathered}
$$


and use known results from semigroup operator methods for solving evolution equations, specifically as presented in [31, Chap. 12.1-2]. The classical initial-value problem gives a unique classical solution if $\hat{C}:=\hat{A}+\hat{B}$ is an infinitesimal generator for a strongly continuous semigroup, $\hat{T}_{m}(t)$. We write of such an operator,

$$
\hat{C} \in \mathcal{G}(M, \omega) \quad \text { iff } \quad\left\|\hat{T}_{m}(t)\right\| \leq M e^{\omega t}, \quad M, \omega \in \mathbb{R} .
$$

Any operator that is skew-adjoint is in $\mathcal{G}(1,0)$. This is true of $\hat{A}$ always, and $\hat{A}+\hat{B}$ iff $m$ is real. If $m$ is not real, we can use the result that if $\hat{A} \in \mathcal{G}(1, \omega)$ and $\hat{B}$ is bounded, with ||$\hat{B}||=|m|$, then $\hat{A}+\hat{B} \in \mathcal{G}(1, \omega+|m|)$, or in this case, $\hat{A}+\hat{B} \in \mathcal{G}(1,|m|)$.

\section{Appendix C: The \pm Components of the Source can be Treated as Supported on Finite Time Individually}

Consider Eq. 58a. Define

$$
{ }_{ \pm} J^{\text {in }}(\Pi ; t):=\int_{\mathbb{R}} d E_{ \pm} \breve{J}(\Pi ; E) \exp \left(-i E\left(t-t_{\text {in }}\right)\right) .
$$

We know that $J^{\text {in }}(\Pi ; t)$ is supported on $t>t_{\text {in }}$. Now define

$$
{ }_{ \pm} J_{2}^{\text {in }}(\Pi ; t)=\left\{\begin{array}{l}
0, \quad t \leq t_{\text {in }} \\
{ }_{ \pm} J^{\text {in }}(\Pi ; t), \quad t>t_{\text {in }},
\end{array}\right.
$$

and call its Fourier transform $\tilde{J}_{2}^{\text {in }}(\Pi ; E)$. Clearly,

$$
\sum_{ \pm} J_{2}^{\text {in }}(\Pi ; t)=\sum_{ \pm} J^{\text {in }}(\Pi ; t)
$$

and hence, by the linearity of the Fourier transform,

$$
\sum_{ \pm} \tilde{J}_{2}^{\text {in }}(\Pi ; E)=\sum_{ \pm} \breve{J}^{\text {in }}(\Pi ; E) .
$$

Consider then the integral we want to evaluate, the first half of the RHS of Eq. 59a,

$$
\begin{aligned}
\psi_{\eta}^{\mathrm{in}}(\Pi ; t) & =\int_{\mathbb{R}} d E g_{\eta, \pm}^{\mathrm{u}}(E, \Pi)\left(\sum_{ \pm} \breve{J}(\Pi ; E) \exp \left(-i E\left(t-t_{\mathrm{in}}\right)\right)\right) \\
& =\int_{\mathbb{R}} d E g_{\eta, \pm}^{\mathrm{u}}(E, \Pi)\left(\sum_{ \pm} \tilde{J}_{2}(E ; \Pi) \exp \left(-i E\left(t-t_{\mathrm{in}}\right)\right)\right) .
\end{aligned}
$$

Therefore we can treat both the \pm components of the source as being supported on $t \leq t_{\text {in }}$, given only the knowledge that their sum is. The argument for the $t \geq t_{\text {out }}$ case proceeds analogously.

Acknowledgements I wish to thank Steven Rose for support and patience. This work is supported by the Engineering and Physical Sciences Research Council. 
Data Availability Not applicable.

Code Availability Not applicable.

\section{Declarations}

Conflict of interest The authors have no conflicts of interest to declare that are relevant to the content of this article.

Open Access This article is licensed under a Creative Commons Attribution 4.0 International License, which permits use, sharing, adaptation, distribution and reproduction in any medium or format, as long as you give appropriate credit to the original author(s) and the source, provide a link to the Creative Commons licence, and indicate if changes were made. The images or other third party material in this article are included in the article's Creative Commons licence, unless indicated otherwise in a credit line to the material. If material is not included in the article's Creative Commons licence and your intended use is not permitted by statutory regulation or exceeds the permitted use, you will need to obtain permission directly from the copyright holder. To view a copy of this licence, visit http://creativecommons.org/licen ses/by/4.0/.

\section{References}

1. Furry, W.H.: On bound states and scattering in positron theory. Phys. Rev. 81(1), 115-124 (1951). https://doi.org/10.1103/PhysRev.81.115

2. Feynman, R.P.: The theory of positrons. Phys. Rev. 76(6), 749-759 (1949). https://doi.org/10. 1103/PhysRev.76.749

3. Gelis, F., Tanji, N.: Schwinger mechanism revisited. Prog. Part. Nucl. Phys. 87, 1-49 (2016). https://doi.org/10.1016/j.ppnp.2015.11.001

4. Schwinger, J.: On gauge invariance and vacuum polarization. Phys. Rev. 82, 664-679 (1951). https://doi.org/10.1103/PhysRev.82.664

5. DeWitt, B.S.: Dynamical Theory of Groups and Fields. Documents on Modern Physics. Gordon and Breach, New York (1965)

6. Barvinsky, A.O., Vilkovisky, G.A.: Phys. Rep. 119(1), 1 (1985) https://doi.org/10.1016/03701573(85)90148-6. https://www.sciencedirect.com/science/article/pii/0370157385901486

7. Camporesi, R.: The generalized Schwinger-Dewitt technique in gauge theories and quantum gravity. Phys. Rep. 196(1), 1-74 (1990) https://doi.org/10.1016/0370-1573(90)90120-Q

8. Dittrich, W., Gies, H.: Probing the Quantum Vacuum: Perturbative Effective Action Approach in Quantum Electrodynamics and Its Application, vol. 166. Springer, Berlin (2000). https://doi.org/ $10.1007 / 3-540-45585-X$

9. Strassler, M.J.: Field theory without Feynman diagrams: one loop effective actions. Nucl. Phys. B 385, 145-184 (1992). https://doi.org/10.1016/0550-3213(92)90098-V

10. Dunne, G.V., Schubert, C.: Worldline instantons and pair production in inhomogenous fields. Phys. Rev. D 72(10), 105004 (2005). https://doi.org/10.1103/PhysRevD.72.105004

11. Dunne, G.V., Wang, Q., Gies, H., Schubert, C.: Worldline instantons and the fluctuation prefactor. Phys. Rev. D 73(6), 065028 (2006) https://doi.org/10.1103/PhysRevD.73.065028

12. Gitman, D.M.: Processes of arbitrary order in quantum electrodynamics with a pair-creating external field. J. Phys. A 10(11), 2007-2020 (1977). https://doi.org/10.1088/0305-4470/10/11/ 026

13. Fradkin, E.S., Gitman, D.M.: Furry picture for quantum electrodynamics with pair-creating external field. Fortschr. Phys. 29(9), 381-411 (1981) https://doi.org/10.1002/prop.19810290902

14. Gavrilov, S.P., Gitman, D.M.: Vacuum instability in external fields. Phys. Rev. D 53(12), 71627175 (1996). https://doi.org/10.1103/physrevd.53.7162 
15. Gavrilov, S.P., Gitman, D.M., Tomazelli, J.L.: Density matrix of a quantum field in a particlecreating background. Nucl. Phys. B 795(3), 645-677 (2008). https://doi.org/10.1016/j.nuclphysb. 2007.11.029

16. Fradkin, E.S., Gitman, D.M., Shvartsman, S.M.: Quantum Electrodynamics with Unstable Vacuum. Springer, Berlin (1991)

17. Gavrilov, S.P., Gitman, D.M.: Proper time and path integral representations for the commutation function. J. Math. Phys. 37, 3118-3130 (1996). https://doi.org/10.1063/1.531559

18. Fanchi, J.: Review of invariant time formulations of relativistic quantum theories. Found. Phys. 23, 487-548 (1993). https://doi.org/10.1007/BF01883726

19. Gieres, F.: Mathematical surprises and Dirac's formalism in quantum mechanics. Rep. Prog. Phys. 63(12), 1893-1931 (2000). https://doi.org/10.1088/0034-4885/63/12/201

20. von Neumann, J.: On rings of operators. Reduction theory. Ann. Math. 50(2), 401-485 (1949). http://www.jstor.org/stable/1969463

21. Hall, B.C.: Quantum Theory for Mathematicians. Graduate Texts in Mathematics. Springer, New York (2013)

22. Mortad, M.H.: Unbounded operators: (square) roots, nilpotence, closability and some related invertibility results (2020). arXiv:2007.12027

23. Blanchard, P., Brüning, E.: Mathematical Methods in Physics. Birkhäuser. Basel (2015). https:// doi.org/10.1007/978-3-319-14045-2

24. Guiggiani, M.: The evaluation of Cauchy principal value integrals in the boundary element method-a review. Math. Comput. Model. 15(3), 175-184 (1991) https://doi.org/10.1016/08957177(91)90063-D

25. Osterwalder, K., Schrader, R.: Axioms for Euclidean Green's functions. Commun. Math. Phys. 31(2), 83-112 (1973). https://doi.org/10.1007/BF01645738

26. Friedlander, F.G., Joshi, M.: Introduction to the Theory of Distributions, 2nd edn. Cambridge University Press, Cambridge (1999)

27. Simon, B.: Essential self-adjointness of Schrödinger operators with positive potentials. Math. Ann. 201, 211-220 (1973). http://eudml.org/doc/162387

28. Reed, M., Simon, B.: I: Functional Analysis. Methods of Modern Mathematical Physics. Academic Press, New York (1981)

29. Rudin, W.: Real and Complex Analysis, 3rd edn. McGraw-Hill, New York (1987)

30. Lesigne, E.: On the behavior at infinity of an integrable function.Am. Math. Mon. 117(2), 175181 (2010)

31. Renardy, M., Rogers, R.C.: An Introduction to Partial Differential Equations. Texts in Applied Mathematics. Springer, New York (2004)

Publisher's Note Springer Nature remains neutral with regard to jurisdictional claims in published maps and institutional affiliations. 